\title{
. \\ X-ray multiphoton-induced Coulomb explosion images complex single molecules
}

\begin{abstract}
Rebecca Boll ${ }^{1,15 凶}$, Julia M. Schäfer ${ }^{2,3,15}$, Benoît Richard ${ }^{2,4,5}$, Kilian Fehre ${ }^{6}$, Gregor Kastirke ${ }^{6}$, Zoltan Jurek ${ }^{2,5}$, Markus S. Schöffler ${ }^{6}$, Malik M. Abdullah, Nils Anders ${ }^{6}{ }^{6}$, Thomas M. Baumann', Sebastian Eckart ${ }^{1}{ }^{6}$, Benjamin Erk ${ }^{(1)}{ }^{7}$, Alberto De Fanis ${ }^{1}$, Reinhard Dörner ${ }^{6}{ }^{6}$, Sven Grundmann ${ }^{6}{ }^{6}$, Patrik Grychtol(1)', Alexander Hartung6, Max Hofmann'6, Markus Ilchen ${ }^{1,8}$, Ludger Inhester (iD) 2,5, Christian Janke ${ }^{6}$, Rui Jin ${ }^{2,9}$, Max Kircher ${ }^{6}{ }^{6}$, Katharina Kubicek ${ }^{1,5}$, Maksim Kunitski ${ }^{6}{ }^{6}$, Xiang Li $^{10}$,

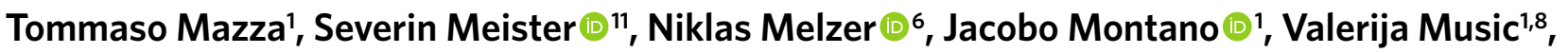
Giammarco Nalin ${ }^{6}{ }^{6}$, Yevheniy Ovcharenko', Christopher Passow ${ }^{7}{ }^{7}$, Andreas Pier ${ }^{6}$, Nils Rennhack $\mathbb{D}^{1}$, Jonas Rist $\mathbb{D}^{6}$, Daniel E. Rivas $\mathbb{1}^{1}$, Daniel Rolles ${ }^{10}{ }^{10}$, Ilme Schlichting ${ }^{10}{ }^{12}$, Lothar Ph. H. Schmidt ${ }^{6}$, Philipp Schmidt ${ }^{1}{ }^{1,8}$, Juliane Siebert ${ }^{6}$, Nico Strenger ${ }^{6}$, Daniel Trabert ${ }^{1}{ }^{6}$, Florian Trinter ${ }^{6,7,13}$, Isabel Vela-Perez ${ }^{6}$, Rene Wagner ${ }^{1}$, Peter Walter ${ }^{14}$, Miriam Weller ${ }^{6}{ }^{6}$, Pawel Ziolkowski ${ }^{1}$, Sang-Kil Son $\mathbb{1}^{2,5}$, Artem Rudenko ${ }^{10}{ }^{10}$, Michael Meyer ${ }^{(1)}$, Robin Santra ${ }^{(1,3,4,5 \bowtie}$ and Till Jahnke ${ }^{1 凶}$

Following structural dynamics in real time is a fundamental goal towards a better understanding of chemical reactions. Recording snapshots of individual molecules with ultrashort exposure times is a key ingredient towards this goal, as atoms move on femtosecond $\left(10^{-15} \mathrm{~s}\right)$ timescales. For condensed-phase samples, ultrafast, atomically resolved structure determination has been demonstrated using $X$-ray and electron diffraction. Pioneering experiments have also started addressing gaseous samples. However, they face the problem of low target densities, low scattering cross sections and random spatial orientation of the molecules. Therefore, obtaining images of entire, isolated molecules capturing all constituents, including hydrogen atoms, remains challenging. Here we demonstrate that intense femtosecond pulses from an $X$-ray free-electron laser trigger rapid and complete Coulomb explosions of 2-iodopyridine and 2-iodopyrazine molecules. We obtain intriguingly clear momentum images depicting ten or eleven atoms, including all the hydrogens, and thus overcome a so-far impregnable barrier for complete Coulomb explosion imaging -its limitation on molecules consisting of three to five atoms. In combination with state-of-the-art multi-coincidence techniques and elaborate theoretical modelling, this allows tracing ultrafast hydrogen emission and obtaining information on the result of intramolecular electron rearrangement. Our work represents an important step towards imaging femtosecond chemistry via Coulomb explosion.
\end{abstract}

\begin{abstract}
n contrast to gas-phase X-ray or electron diffraction ${ }^{1-7}$, Coulomb explosion imaging (CEI) is an experimental technique that is equally sensitive to light and heavy atoms, providing information in momentum space, typically in a molecular frame of reference. It is inherently tailored to study single molecules and has originally been performed by sending beams of ionized molecules through thin foils ${ }^{8}$, thus stripping off electrons. The key to the success of CEI is a rapid charge up of all the atoms, triggering an explosion of molecules due to Coulomb repulsion. The fast removal of sufficiently many electrons is important to ensure that the molecule fragments into atomic ions without undergoing structural rearrangement during the charge up. The formation of long-lived intermediate molecular fragment ions would complicate the mapping from asymptotic
\end{abstract}

fragment momenta to the initial atomic positions ${ }^{9}$. CEI is ideally suited to separately address all the atoms in a molecule, thus providing site-specific information on the entire molecule. Moreover, it has the potential of imaging the complete (square of the) nuclear wavefunction ${ }^{8}$.

Nowadays, CEI is typically triggered through photoionization, which opens the door to time-resolved experiments. For example, intense, femtosecond laser pulses allowed retrieving the complete three-dimensional (3D) fragmentation pattern of a small molecule with five atoms ${ }^{10}$ or isolating a rare roaming reaction ${ }^{11}$. Inner-shell absorption of an X-ray photon can-depending on the photon energy and atomic species-also create multiple charges on a molecule due to Auger cascades. However, among the many studies on

\footnotetext{
'European XFEL, Schenefeld, Germany. ${ }^{2}$ Center for Free-Electron Laser Science CFEL, Deutsches Elektronen-Synchrotron DESY, Hamburg, Germany. ${ }^{3}$ Department of Chemistry, Universität Hamburg, Hamburg, Germany. ${ }^{4}$ Department of Physics, Universität Hamburg, Hamburg, Germany. ${ }^{5}$ The Hamburg Centre for Ultrafast Imaging, Hamburg, Germany. ${ }^{6}$ Institut für Kernphysik, J. W. Goethe-Universität, Frankfurt am Main, Germany. ${ }^{7}$ Deutsches Elektronen-Synchrotron DESY, Hamburg, Germany. ${ }^{8}$ Institut für Physik und CINSaT, Universität Kassel, Kassel, Germany. ${ }^{9}$ Department of Physics and Astronomy, Shanghai Jiao Tong University, Shanghai, China. ${ }^{10}$ J.R. Macdonald Laboratory, Department of Physics, Kansas State University, Manhattan, KS, USA. " Max-Planck-Institut für Kernphysik, Heidelberg, Germany. ${ }^{12}$ Max-Planck-Institut für medizinische Forschung, Heidelberg, Germany. ${ }^{13}$ Molecular Physics, Fritz-Haber-Institut der Max-Planck-Gesellschaft, Berlin, Germany. ${ }^{14}$ SLAC National Accelerator Laboratory, Menlo Park, CA, USA. ${ }^{15}$ These authors contributed equally: Rebecca Boll, Julia M. Schäfer.凶e-mail: rebecca.boll@xfel.eu; robin.santra@cfel.de; till.jahnke@xfel.eu
} 

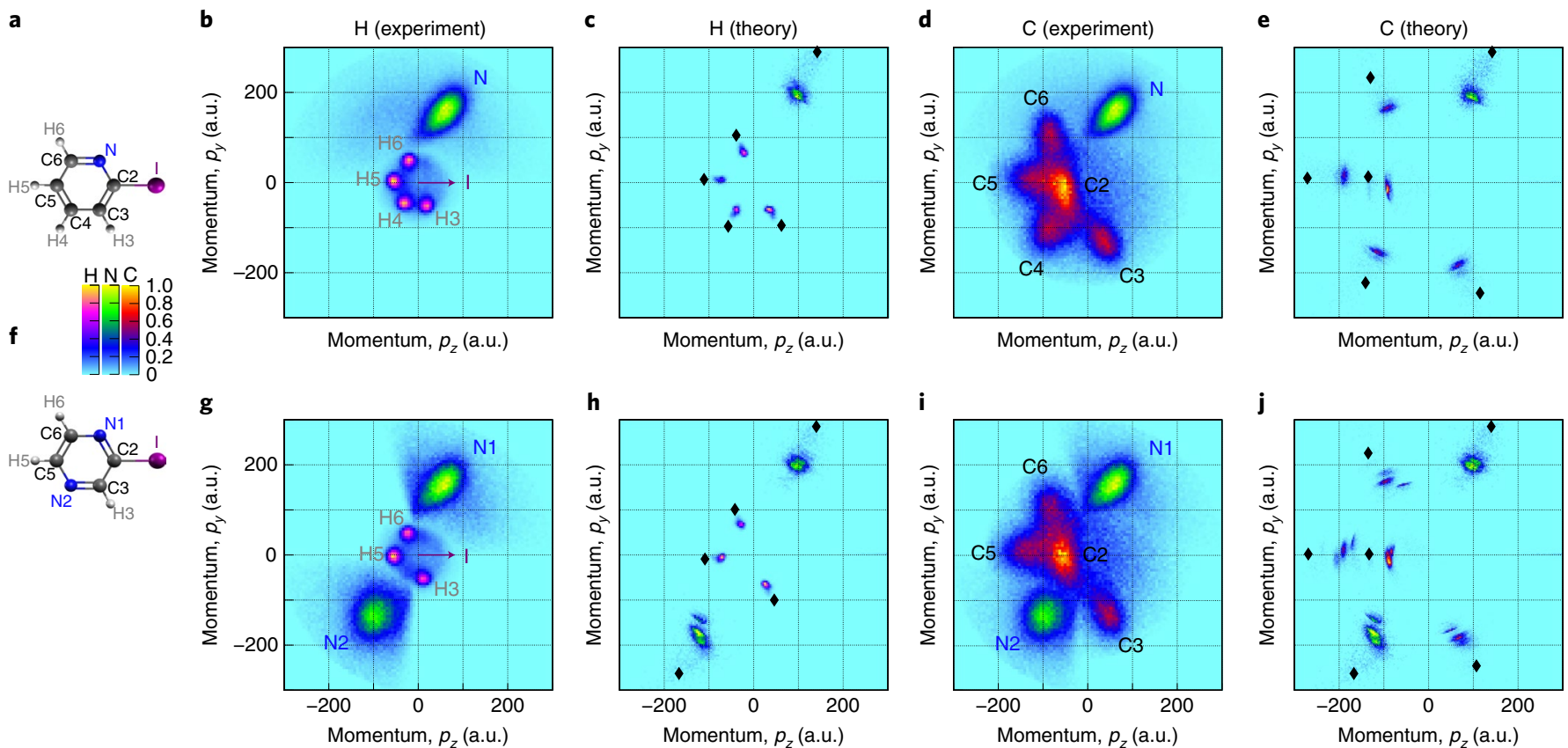

h

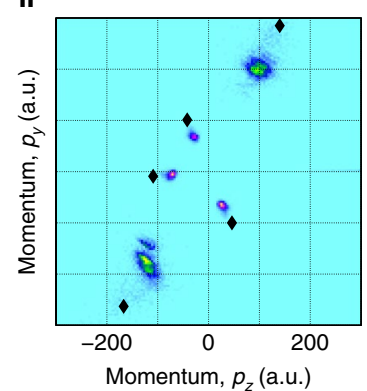

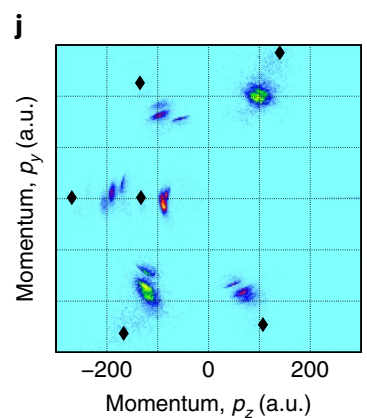

Fig. 1 | CEl of 2-iodopyridine and 2-iodopyrazine. a, Newton plots of $\mathrm{N}^{+}$and $\mathrm{H}^{+}\left(\mathbf{b}\right.$ and $\mathbf{g}$ ) and $\mathrm{N}^{+}$and $\mathrm{C}^{+}$( $\mathbf{d}$ and $\mathbf{i}$ ) show the measured absolute momenta following Coulomb explosion of 2-iodopyridine $\left(\mathrm{C}_{5} \mathrm{H}_{4} \mathrm{IN}\right)(\mathbf{a})$ and 2-iodopyrazine $\left(\mathrm{C}_{4} \mathrm{H}_{3} \mathrm{IN}_{2}\right)(\mathbf{f})$ molecules. For all the shots in which $\mathrm{I}^{+}, \mathrm{N}^{+}$, and at least one $\mathrm{H}^{+}$and one $\mathrm{C}^{+}$were detected, the coordinate frame is rotated such that the $\mathrm{I}^{+}$momentum points along the $z$ axis $\left(p_{\mathrm{I}_{x}}=p_{\mathrm{l}_{y}}=0\right.$ ) and the $\mathrm{N}^{+}$momentum spans the $y-z$ plane together with the $I^{+}$momentum $\left(p_{N_{x}}=0, p_{N_{y}}>0\right)$. The momentum of the third particle is plotted in this coordinate frame. No background was subtracted. The corresponding modelling results from XMDYN are shown in $\mathbf{c}, \mathbf{e}, \mathbf{h}$ and $\mathbf{j}$. The diamond-shaped markers depict the asymptotic momenta when a single positive charge is instantaneously applied to each atom.

3D momentum imaging using X-ray ionization, only a few of them have studied somewhat larger molecules up to now ${ }^{12,13}$.

With the development of X-ray free-electron lasers (XFELs) offering pulse duration of a few femtoseconds, the achievable intensity has increased by orders of magnitude. This facilitated, for the first time, multiphoton inner-shell absorption, removing many electrons from a single atom within one X-ray pulse ${ }^{14}$. Several XFEL experiments have obtained 3D momentum distributions of small molecules with four to five atoms ${ }^{15,16}$, and pioneering work touched the frontier of imaging larger molecules ${ }^{17,18}$. The first time-resolved experiments have investigated processes such as dissociation ${ }^{19}$, vibration $^{20}$, isomerization reactions ${ }^{21,22}$ and charge transfer ${ }^{23}$ with CEI. However, CEI of complex molecules was considered unfeasible, because it was expected that recording all the molecular constituents in coincidence would be necessary-a requirement, up to now, impossible to fulfil for more than a few atoms with sufficient statistics, as the detection efficiency of each ion is typically only about $60 \%$.

Here we demonstrate that intense, femtosecond soft X-ray pulses from a high-repetition-rate free-electron laser, the European XFEL, make it possible to image a complex molecule in its entirety, as well as to obtain detailed information on the ultrafast intramolecular electron rearrangement without measuring all the fragments in coincidence. The experiment was carried out using a cold-target recoil ion momentum spectroscopy (COLTRIMS) reaction microscope $^{24}$ that is part of the Small Quantum Systems (SQS) scientific instrument at the European $\mathrm{XFEL}^{25}$. 2-Iodopyridine $\left(\mathrm{C}_{5} \mathrm{H}_{4} \mathrm{IN}\right)$ or 2-iodopyrazine $\left(\mathrm{C}_{4} \mathrm{H}_{3} \mathrm{IN}_{2}\right)$ molecules were prepared in a dilute gas jet through supersonic expansion, such that each X-ray pulse typically interacted with only a single molecule. The X-ray focus size was $\sim 1.4 \mu \mathrm{m}$ and the photon energy was set to $2 \mathrm{keV}$; the average pulse energy was $1 \mathrm{~mJ}$. The 3D momenta of the ionized fragments were measured in coincidence. More details are given in Methods.
Figure 1 shows the ion momenta resulting from the fragmentation of molecules into $\mathrm{I}^{+}, \mathrm{N}^{+}, \mathrm{C}^{+}$and $\mathrm{H}^{+}$. The molecular frame of reference is defined by the $\mathrm{I}^{+}$and $\mathrm{N}^{+}$momenta; the ion species, identified by their different mass-to-charge ratios, are plotted in different colours. The structural analogies between the equilibrium molecular geometries and momentum distributions are very clear. In particular, the hydrogen $(\mathrm{H} 3-\mathrm{H} 6)$ signals are well localized in Fig. 1, but the momenta of the individual carbon atoms (C2-C6) can become similarly separated (Fig. 3). As far as we are aware, momentum images of this quality are unprecedented for such complex molecules. We attribute the clarity predominantly to the fast charging of all ten or eleven atoms within only a few femtoseconds (Fig. 2d), leading to a direct explosion without the rotation or formation of intermediate molecular fragments.

We emphasize that the Newton plots in Fig. 1 display coincidences between only three ions (out of sets of four measured ions; Methods). If only a fraction of the generated ion fragments is detected in coincidence, this usually implies a reduction of information. Due to the high repetition rate of the European XFEL (here up to $570 \mathrm{X}$-ray pulses per second), we could also record up to eightfold ion coincidences, which produce identical-looking images in this case (Extended Data Fig. 1). This reveals that for the studied planar molecules, the reduced set of information from partial coincidences captures the essence of the asymptotic momentum density distribution of the fully fragmented molecule. The sharp momentum distributions (Fig. 1) indicate that the entire fragmentation pathway is determined by the final charges of only very few atomic fragments. Therefore, we obtain molecular fingerprints in the momentum distributions that provide a direct insight into the fragmentation dynamics.

The carbon atom located closest to the iodine atom (C2; Fig. 1d) receives only little overall momentum, because it is repelled by the heavy iodine ion, as well as the other ions in the ring. The $\mathrm{C} 2$ ion, 
a
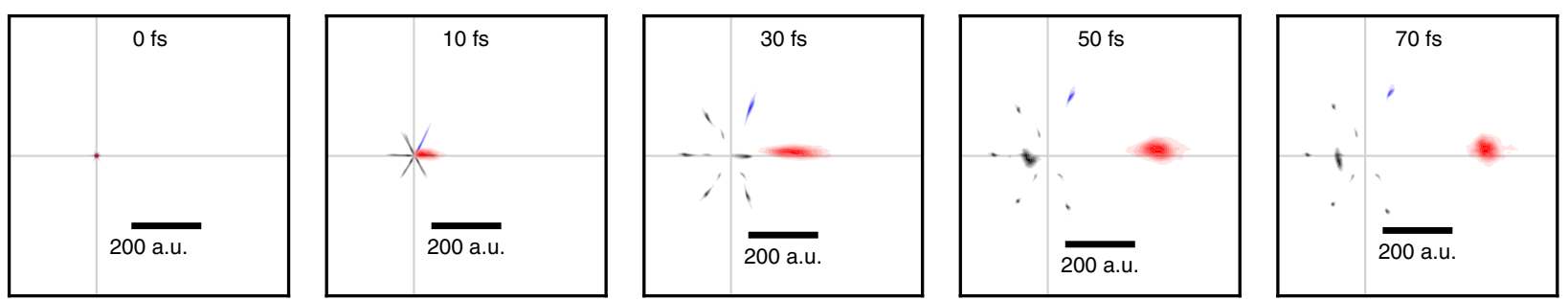

b
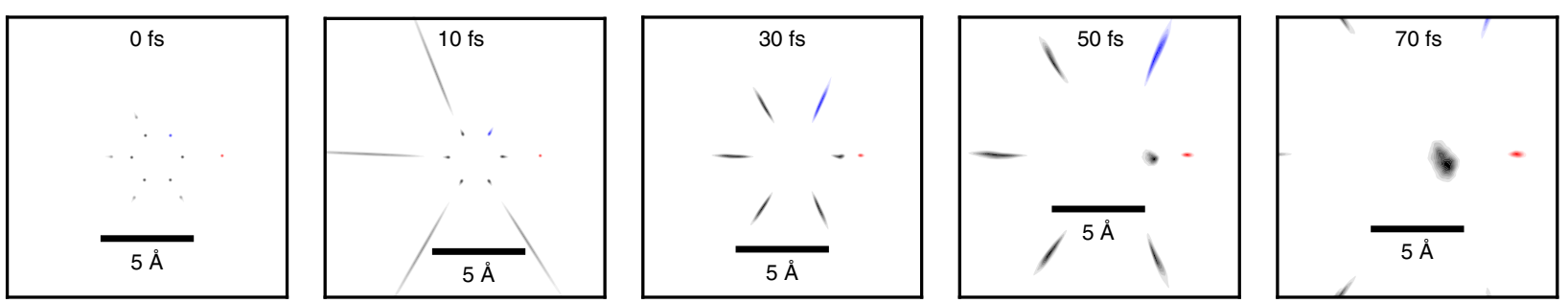

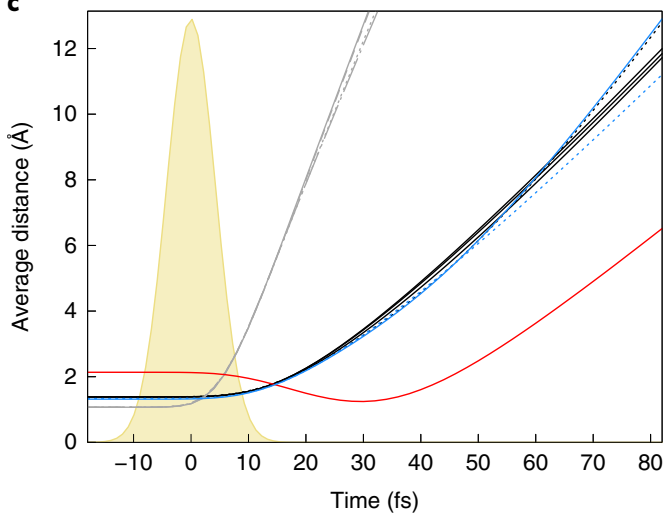

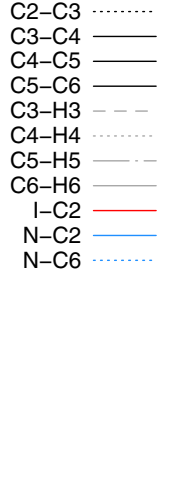

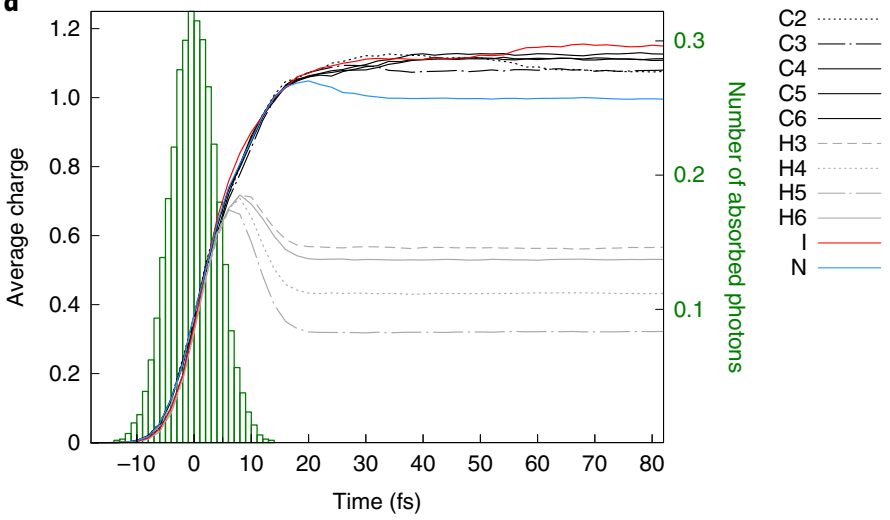

Fig. 2 | Time-dependent geometry and charge evolution of 2-iodopyridine molecules, created from calculated XMDYN trajectories yielding $\mathbf{I}^{+}+\mathrm{N}^{+}$. a,b, Snapshots of the distributions of ion momenta (a) and ion positions ( $\mathbf{b}$ ) at different times (red, $\mathrm{I}^{+}$; blue, $\mathrm{N}^{+}$; black, $\mathrm{C}^{+}$; grey, $\mathrm{H}^{+}$). The origin of the coordinate frame is the molecular centre of mass, the horizontal axis points along the equilibrium position of I, and the plane of the figure is spanned by the I and $\mathrm{N}$ equilibrium positions such that $\mathrm{N}$ points upwards. The grey gridlines indicate $p_{x}=p_{z}=0$. $\mathbf{c}, \mathbf{d}$, Average interatomic distances (c) and average charges (d). The yellow area in c indicates the assumed X-ray pulse shape, namely, a Gaussian with a full-width at half-maximum of 10 fs (Extended Data Fig. 2 shows the results for the 20 fs case). In d, the average number of absorbed photons per molecule and per femtosecond are shown in green.

therefore, remains almost at rest after fragmentation (Supplementary Videos 1 and 2). Furthermore, the proton momenta (Fig. 1b,g) are unexpectedly small ( $\left|p_{\mathrm{H}}\right| \approx 55$ a.u.). A simple point-charge Coulomb explosion that instantaneously assigns a charge of +1 to each atom at its equilibrium position results in proton momenta of $\left|p_{H}\right| \approx 110$ a.u. (Fig. 1c,h, black markers). The lower observed momenta, therefore, imply rapid proton ejection before the full charge up of the molecule. More advanced theoretical modelling is clearly essential for extracting all the information from the measured momenta.

We employed the XMDYN theoretical toolkit ${ }^{26}$, which combines atomic ab initio X-ray physics with molecular dynamics in a Monte Carlo scheme. It includes charge transfer between the atom pairs based on a classical over-the-barrier model. The resulting Newton plots are shown in Fig. 1c,e,h,j, and further computational details can be found in Methods. The overall agreement with the experimental data is surprisingly good considering that for computational efficiency, no molecular orbitals or chemical bonds are included in the modelling. The demonstrated ability to reproduce experimental ion momenta by inserting a known equilibrium molecular geometry into our model represents the first important step towards future structural inversions of experimental XFEL CEI data. The comparison with the experiment also helped to improve our model
(Methods). We mainly ascribe the remaining discrepancies between experiment and theory (Fig. 1) to residual chemical bonding. This can probably be overcome in the future by adding a force field in the model. An iterative optimization scheme would compare the calculated and experimentally measured momenta, thus identifying the start geometry that leads to the best match. Although not a high-precision method, such a procedure could be used, for example, to reconstruct the $\mathrm{D}_{2} \mathrm{O}$ molecule from CEI data with an accuracy of $0.3 \AA$ for the bond length and $15^{\circ}$ for the bond angle ${ }^{27}$.

Our modelling provides access to the structural dynamics occurring on femtosecond timescales. Figure $2 \mathrm{a}, \mathrm{b}$ shows snapshots of the distributions of ion momenta and positions during and after the $\mathrm{X}$-ray pulse. Figure $2 \mathrm{c}$, d displays the average interatomic distances and average atomic charges as a function of time. Hydrogens exhibit the earliest onset of evolving dynamics, already starting during the $\mathrm{X}$-ray pulse. The average $\mathrm{C}-\mathrm{H}$ distance increases by almost a factor of two within the first $5 \mathrm{fs}$ after the pulse centre, confirming the rapid proton ejection. Such a behaviour was also predicted for hydride clusters ${ }^{28}$. The heavier fragments only start moving notably after the $\mathrm{X}$-ray pulse is over. Although the distances of the $\mathrm{C}-\mathrm{C}$ and $\mathrm{C}-\mathrm{N}$ bonds elongate as expected, the I-C2 distance first decreases drastically by almost a factor of two, reaching a minimum after $30 \mathrm{fs}$. 
a

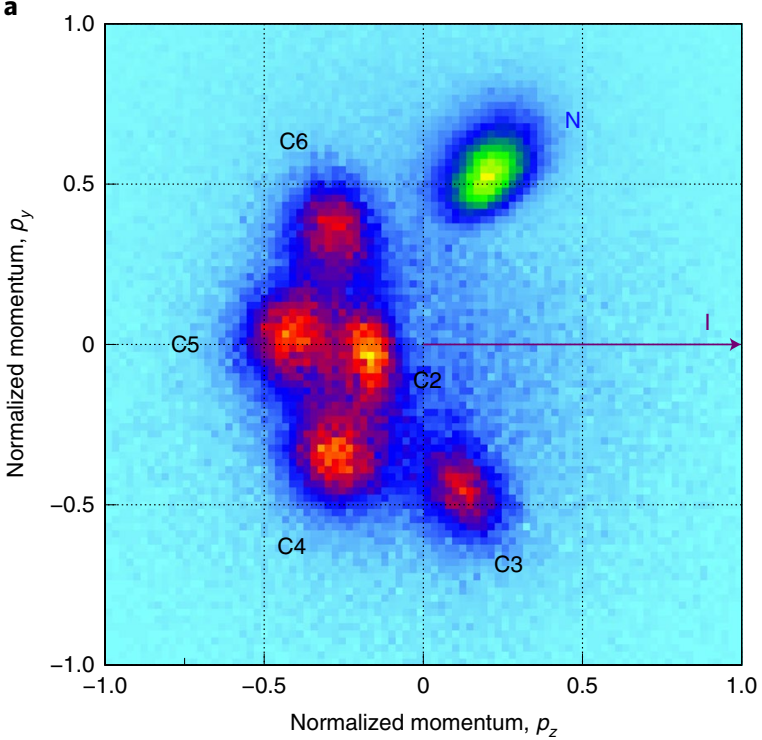

b

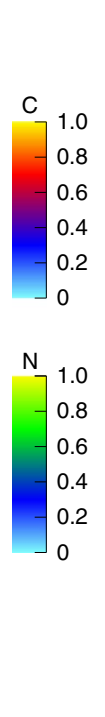

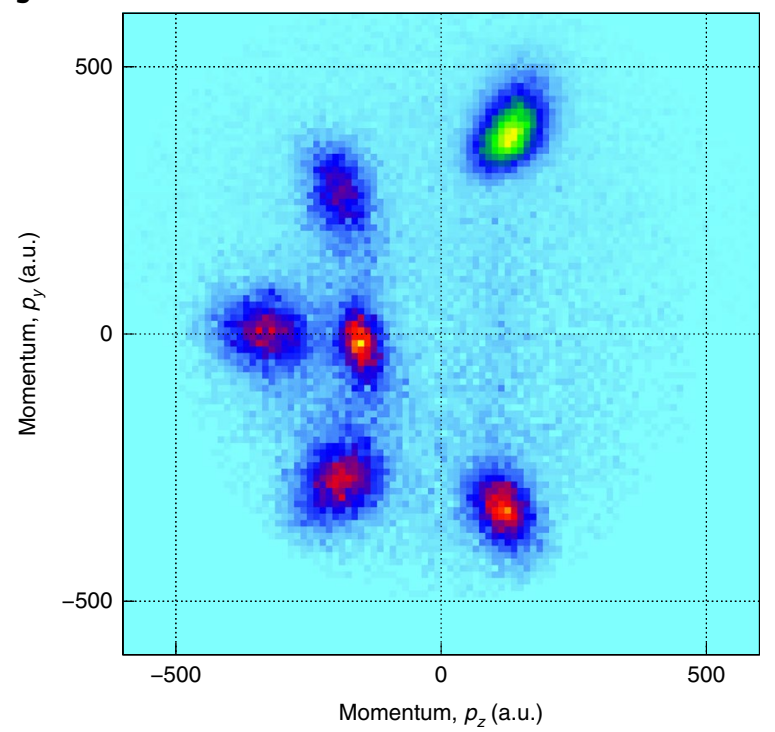

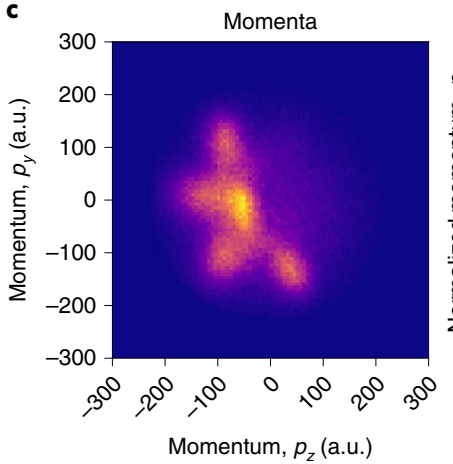

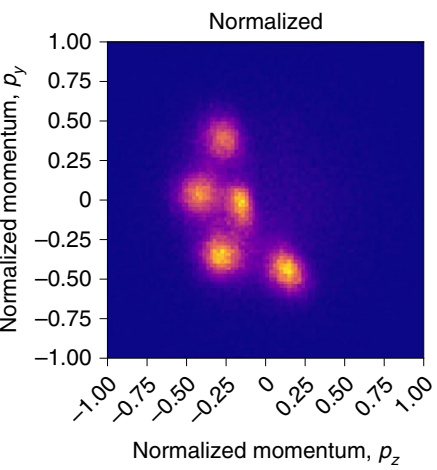
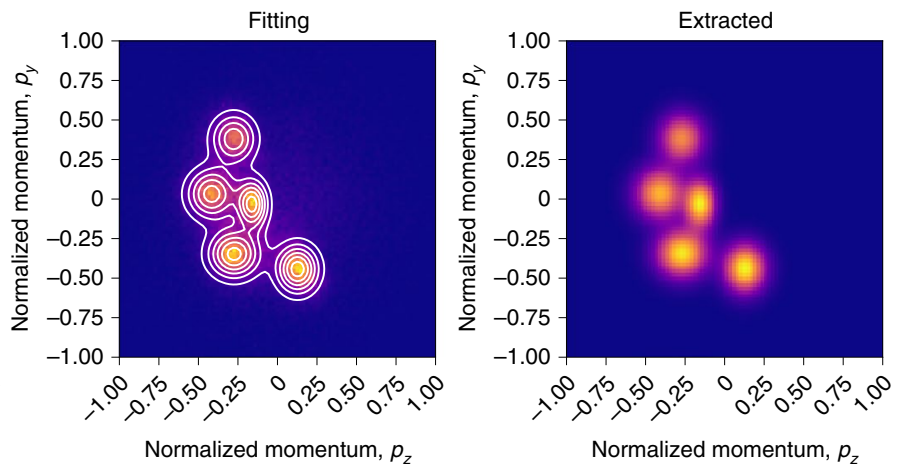

Fig. 3 | Momentum-space normalization and extraction of site-specific charge abundances. a, Normalized Newton plot for $\mathrm{I}^{+}, \mathrm{N}^{+}$and $\mathrm{C}^{+}$coincidences (compare with Fig. 1d). b, Absolute molecular-frame momenta for $\mathrm{I}^{4+}, \mathrm{N}^{2+}$ and $\mathrm{C}^{2+}$ coincidences. c, Visualization of the procedure for extracting site-specific charge abundances.

It only starts to become larger than the equilibrium distance after about $50 \mathrm{fs}$. This intriguing behaviour is caused by the force acting on $\mathrm{C} 2$ that is initially directed towards iodine, until the ring has undergone Coulomb explosion and the repulsion by iodine dominates. The minimum of the I-C2 distance is also present for channels with a higher total charge, for example, in the $\mathrm{I}^{4+}+\mathrm{N}^{2+}$ coincidence, where it is reached after 13 fs (Extended Data Fig. 3). In Fig. 2d, all the average charges first rise simultaneously. Although the I, C and $\mathrm{N}$ charges reach a plateau after $20 \mathrm{fs}$, the hydrogen charges exhibit a maximum after $10 \mathrm{fs}$, because from 10 to $14 \mathrm{fs}$, electron transfer is only possible from $\mathrm{C}$ to $\mathrm{H}^{+}$but not from $\mathrm{H}$ to $\mathrm{C}^{+}$due to the respective barriers in the model (Extended Data Fig. 4). The final charge states are determined by the late-time charge evolution (Extended Data Fig. 5), and the calculated average total molecular charge is +9 for fragmentation into $\mathrm{I}^{+}$and $\mathrm{N}^{+}$.

At $2 \mathrm{keV}$ photon energy, $91 \%$ of all the photoionization events take place at the iodine site (Extended Data Table 1), and the experimental data contain iodine ions with charges up to $\mathrm{I}^{25+}$. However, we also detect $\mathrm{H}^{+}, \mathrm{C}^{+}-\mathrm{C}^{4+}$ and $\mathrm{N}^{+}-\mathrm{N}^{3+}$, indicating that ultrafast electron rearrangement occurs within the molecule-a key process governing the structural dynamics and an active topic of investigation in several fields. To trace the result of the intramolecular electron transfer, that is, the final charges of the individual atoms, we can obtain site-specific charge-state distributions. The blurred carbon distributions (Fig. 1d) make such an assignment difficult, however, normalizing the carbon and nitrogen momenta by the magnitude of the corresponding iodine momentum $\left(p_{\text {norm }}=p /\left|p_{\mathrm{I}}\right|\right)$ separates the distributions (Fig. 3a). We also note that the Newton plots for higher molecular charges provide clearly distinct carbon-ion distributions even without normalization, as shown in Fig. $3 b$ for the $\mathrm{I}^{4+}, \mathrm{N}^{2+}$ and $\mathrm{C}^{2+}$ channel (see also Extended Data Fig. 6 for other charge states). A higher final charge implies a quicker charge up, thus approximating an instantaneous Coulomb explosion better.

For extracting site-specific charge-state distributions, we employ a multiple two-dimensional (2D) Gaussian fit to the normalized Newton plots (Fig. 3, bottom row, and Methods). Figure $4 \mathrm{a}-\mathrm{c}$ displays the resulting experimental carbon charge-state distributions for each position in the ring and for different coincidence channels. The average carbon charge shifts to higher values for higher iodine charges. Moreover, although the abundances of carbon charge states are uniformly distributed over the ring for the $\mathrm{I}^{+}+\mathrm{N}^{+}$case (Fig. 4a), the $\mathrm{C} 2$ atom (red bar) is more often singly charged and less often triply charged than the other atoms for higher molecular charges (Fig. 4b,c). This may seem counterintuitive, because the C2 atom is located closest to the source of the charge (iodine) and in contrast to the other atoms, it stays within close vicinity of the iodine for several tens of femtoseconds (Fig. 2c). A shorter internuclear distance strongly increases the electron-transfer probability ${ }^{23}$. However, the trend in the charge-state distribution shows that these effects are overcompensated by the rapid rearrangement of electrons 

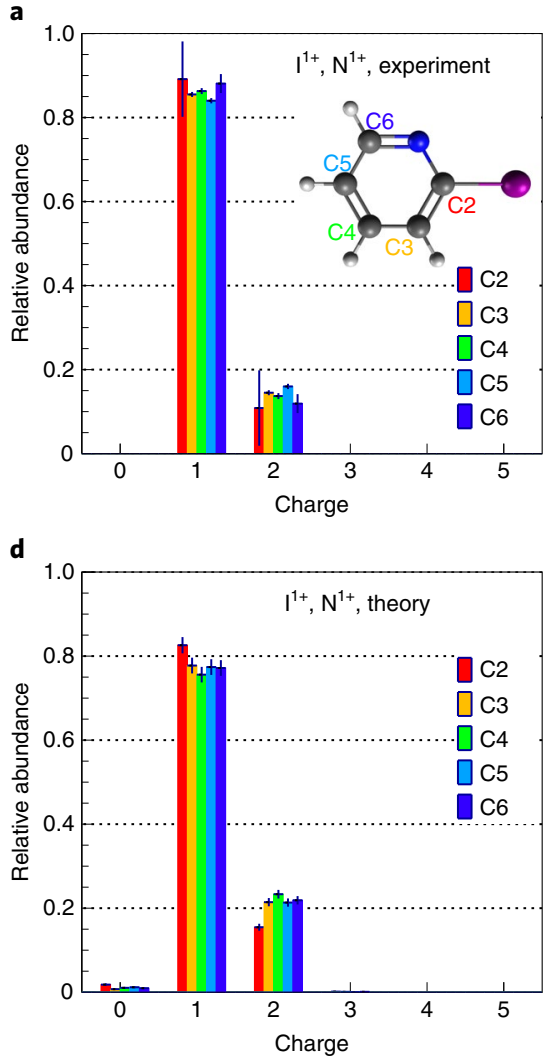
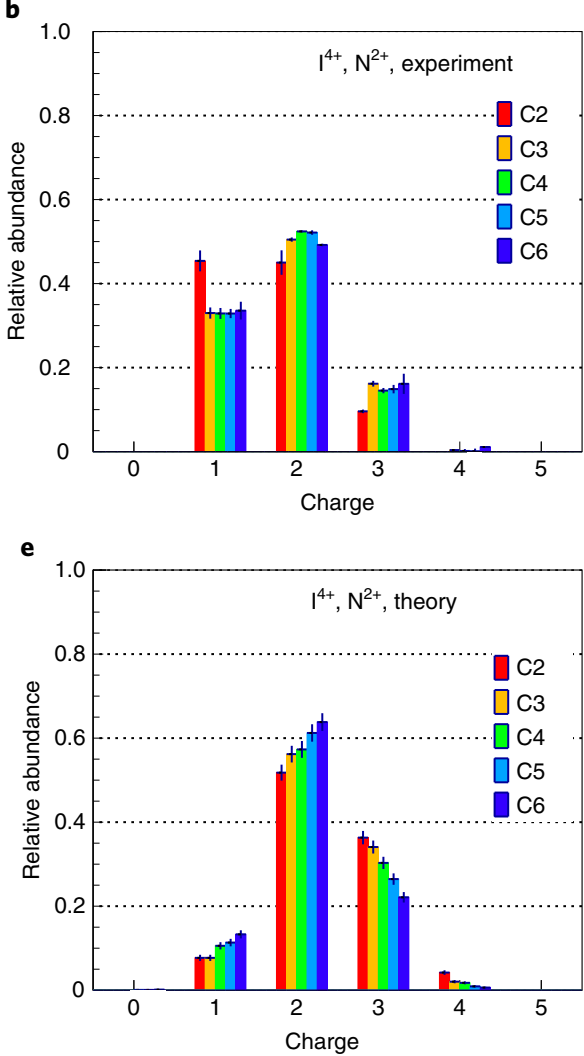
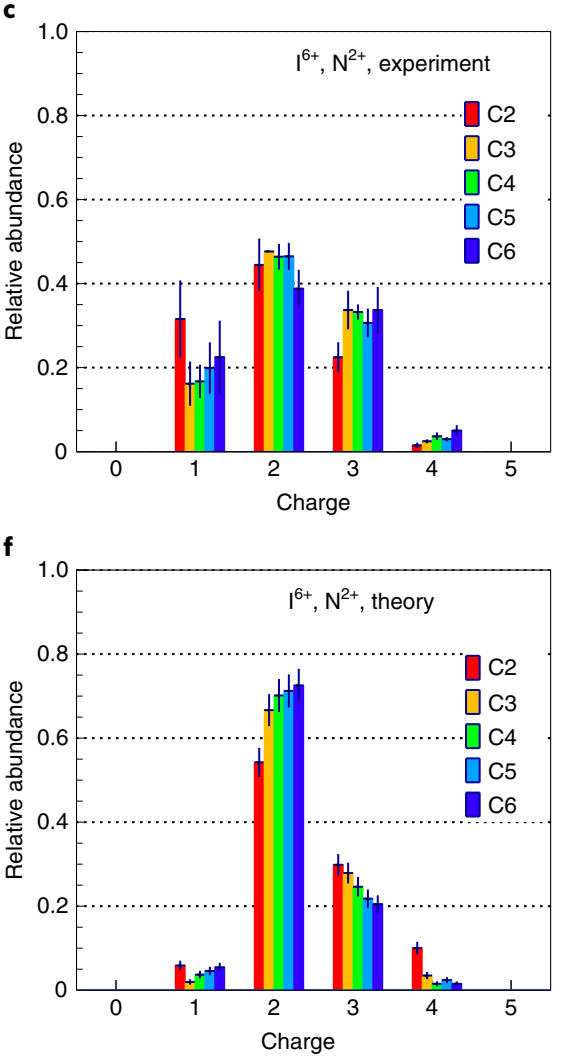

Fig. 4 | Charge-state distributions of carbon atoms for different coincidence channels of 2-iodopyridine. a-c, Experimental ion yields are obtained by fitting a 2D Gaussian to each peak in the respective Newton plots (Fig. 3). The experimental error bars represent an estimate of the systematic error introduced in the fitting (Methods). The experiment is insensitive to neutral fragments. d-f, Corresponding theory results are shown with the statistical error derived from the finite number of trajectories. The fluences used in the calculations are $0.75 \times 10^{11}(\mathbf{d}), 1.80 \times 10^{11}(\mathbf{e})$ and $2.30 \times 10^{11}(\mathbf{f})$ photons $\mu \mathrm{m}^{-2}$.

on timescales much faster than nuclear motion, adapting to the momentary geometry. Coulomb repulsion causes the positive partial charge to preferentially locate furthest away from the highly charged iodine atom, thus pulling the electron density towards the attractive potential of the iodine ion, as suggested previously ${ }^{29,30}$. The average charge is reproduced well in the calculations (Fig. $4 \mathrm{~d}-\mathrm{f}$ ). However, the lower charge on the $\mathrm{C} 2$ atom for the higher charge coincidences is not captured by the current model and the overall distribution matches only for the $\mathrm{I}^{+}+\mathrm{N}^{+}$coincidence channel.

Our results demonstrate that Coulomb explosion induced by intense, femtosecond X-ray pulses can, under certain conditions, image complex molecules in their entirety, particularly including all the hydrogen atoms. Its scalability to non-planar and more complex molecules, such as samples without marker atoms, remains to be tested in future experiments. The key advantage of using an XFEL to trigger the explosion lies in the ease of creating very high charge states within a few femtoseconds, which is very difficult to achieve with femtosecond optical lasers. The ability to identify all the atoms in the momentum maps allowed us to retrieve the result of the intramolecular electron rearrangement at each atomic site. Advanced theoretical modelling added an insight into the ionization dynamics and geometry evolution of the fragments. In addition, the comparison with experimental data initiated theory developments necessary on the route towards structural inversion. We could show that partial ion coincidences of complex molecules with a well-defined structure can offer an unexpected degree of information. The method will further benefit from even shorter pulse durations and steadily increasing repetition rates of XFELs, which will enable the detection of even higher many-fold ion coincidences. The capability to reveal higher-order correlations renders the combination of XFEL and CEI a promising atomic-resolution imaging tool. In diffraction-based methodologies, typically, only second-order correlations are accessible. Although both ultrafast X-ray and electron diffraction hold the prospect to measure hydrogen atoms according to simulations ${ }^{31}$, a direct experimental demonstration in the gas phase is still pending and will require future improvements in the data quality ${ }^{31}$. Possible future advances of XFEL-induced CEI include its application as a probe step in the monitoring of atomic motion in time-resolved pump-probe experiments, as well as employing multiple FEL pulses of different colors, targeted to ionize specific atoms or the entire molecule. The unprecedentedly clear experimental momentum distributions, in combination with a powerful theoretical toolkit, are thus very encouraging with regard to recording videos of photochemical reactions.

\section{Online content}

Any methods, additional references, Nature Research reporting summaries, source data, extended data, supplementary information, acknowledgements, peer review information; details of author contributions and competing interests; and statements of data and code availability are available at https://doi.org/10.1038/ s41567-022-01507-0.

Received: 7 November 2021; Accepted: 6 January 2022; Published online: 21 February 2022

\section{References}

1. Küpper, J. et al. X-ray diffraction from isolated and strongly aligned gas-phase molecules with a free-electron laser. Phys. Rev. Lett. 112, 083002 (2014). 
2. Minitti, M. P. et al. Imaging molecular motion: femtosecond X-ray scattering of an electrocyclic chemical reaction. Phys. Rev. Lett. 114, 255501 (2015).

3. Stankus, B. et al. Ultrafast X-ray scattering reveals vibrational coherence following Rydberg excitation. Nat. Chem. 11, 716-721 (2019).

4. Hensley, C. J., Yang, J. \& Centurion, M. Imaging of isolated molecules with ultrafast electron pulses. Phys. Rev. Lett. 109, 133202 (2012).

5. Yang, J. et al. Imaging $\mathrm{CF}_{3} \mathrm{I}$ conical intersection and photodissociation dynamics with ultrafast electron diffraction. Science 361, 64-67 (2018).

6. Wolf, T. J. A. et al. The photochemical ring-opening of 1,3-cyclohexadiene imaged by ultrafast electron diffraction. Nat. Chem. 11, 504-509 (2019).

7. Wolter, B. et al. Ultrafast electron diffraction imaging of bond breaking in di-ionized acetylene. Science 354, 308-312 (2016).

8. Vager, Z., Naaman, R. \& Kanter, E. P. Coulomb explosion imaging of small molecules. Science 244, 426-431 (1989).

9. Zhou, W. et al. Coulomb explosion imaging for gas-phase molecular structure determination: an ab initio trajectory simulation study. J. Chem. Phys. 153, 184201 (2020)

10. Pitzer, M. et al. Direct determination of absolute molecular stereochemistry in gas phase by Coulomb explosion imaging. Science 341, 1096-1100 (2013).

11. Endo, T. et al. Capturing roaming molecular fragments in real time. Science 370, 1072-1077 (2020)

12. Pitzer, M. et al. Absolute configuration from different multifragmentation pathways in light-induced Coulomb explosion imaging. ChemPhysChem 17, 2465-2472 (2016).

13. Ablikim, U. et al. Isomer-dependent fragmentation dynamics of inner-shell photoionized difluoroiodobenzene. Phys. Chem. Chem. Phys. 19 13419-13431 (2017)

14. Young, L. et al. Femtosecond electronic response of atoms to ultra-intense X-rays. Nature 466, 56-61 (2010)

15. Erk, B. et al. Ultrafast charge rearrangement and nuclear dynamics upon inner-shell multiple ionization of small polyatomic molecules. Phys. Rev. Lett. 110, 053003 (2013)

16. Rudenko, A. et al. Femtosecond response of polyatomic molecules to ultra-intense hard X-rays. Nature 546, 129-132 (2017).

17. Kukk, E., Motomura, K., Fukuzawa, H., Nagaya, K. \& Ueda, K. Molecular dynamics of XFEL-induced photo-dissociation, revealed by ion-ion coincidence measurements. Appl. Sci. 7, 531 (2017)

18. Nagaya, K. et al. Ultrafast dynamics of a nucleobase analogue illuminated by a short intense X-ray free electron laser pulse. Phys. Rev. X 6, 021035 (2016).

19. Schnorr, K. et al. Electron rearrangement dynamics in dissociating $\mathrm{I}_{2}^{n+}$ molecules accessed by extreme ultraviolet pump-probe experiments. Phys. Rev. Lett. 113, 073001 (2014).
20. Jiang, $Y$. H. et al. Investigating two-photon double ionization of $\mathrm{D}_{2}$ by XUV-pump-XUV-probe experiments. Phys. Rev. A 81, 051402(R) (2010).

21. Liekhus-Schmaltz, C. E. et al. Ultrafast isomerization initiated by X-ray core ionization. Nat. Commun. 6, 8199 (2015).

22. Jiang, Y. H. et al. Ultrafast extreme ultraviolet induced isomerization of acetylene cations. Phys. Rev. Lett. 105, 263002 (2010)

23. Erk, B. et al. Imaging charge transfer in iodomethane upon X-ray photoabsorption. Science 345, 288-291 (2014).

24. Ullrich, J. et al. Recoil-ion and electron momentum spectroscopy: reaction-microscopes. Rep. Prog. Phys. 66, 1463 (2003).

25. Decking, W. et al. A MHz-repetition-rate hard X-ray free-electron laser driven by a superconducting linear accelerator. Nat. Photon. 14, 391-397 (2020).

26. Jurek, Z., Son, S.-K., Ziaja, B. \& Santra, R. XMDYN and XATOM: versatile simulation tools for quantitative modeling of X-ray free-electron laser induced dynamics of matter. J. Appl. Cryst. 49, 1048-1056 (2016).

27. Légaré, F. et al. Laser Coulomb-explosion imaging of small molecules. Phys. Rev. A 71, 013415 (2005)

28. Di Cintio, P., Saalmann, U. \& Rost, J.-M. Proton ejection from molecular hydride clusters exposed to strong X-ray pulses. Phys. Rev. Lett. 111, 123401 (2013).

29. Hao, Y., Inhester, L., Son, S.-K. \& Santra, R. Theoretical evidence for the sensitivity of charge-rearrangement-enhanced X-ray ionization to molecular size. Phys. Rev. A 100, 013402 (2019).

30. Amini, K. et al. Alignment, orientation, and Coulomb explosion of difluoroiodobenzene studied with the pixel imaging mass spectrometry (PImMS) camera. J. Chem. Phys. 147, 013933 (2017).

31. Ma, L. et al. Ultrafast X-ray and electron scattering of free molecules: a comparative evaluation. Struct. Dyn. 7, 034102 (2020).

Publisher's note Springer Nature remains neutral with regard to jurisdictional claims in published maps and institutional affiliations.

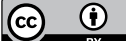

Open Access This article is licensed under a Creative Commons Attribution 4.0 International License, which permits use, sharing, adaptation, distribution and reproduction in any medium or format, as long as you give appropriate credit to the original author(s) and the source, provide a link to the Creative Commons license, and indicate if changes were made. The images or other third party material in this article are included in the article's Creative Commons license, unless indicated otherwise in a credit line to the material. If material is not included in the article's Creative Commons license and your intended use is not permitted by statutory regulation or exceeds the permitted use, you will need to obtain permission directly from the copyright holder. To view a copy of this license, visit http://creativecommons. org/licenses/by/4.0/.

(c) The Author(s) 2022 


\section{Methods}

The experiment was performed using a cold-target recoil ion momentum spectroscopy (COLTRIMS) reaction microscope ${ }^{24}$ that is available for users as part of the SQS scientific instrument at the European XFEL. The simulations were carried out with an extended version ${ }^{32,33}$ of the $\mathrm{XMDYN}^{26}$ toolkit that models the dynamics of matter exposed to high-intensity X-rays.

X-ray pulse properties. The European XFEL, operated at a base repetition rate of $10 \mathrm{~Hz}$, provides bursts of electron pulses with a spacing of $1.1 \mathrm{MHz}$, out of which we used every sixth pulse for generating $\mathrm{X}$-ray pulses for our experiment, that is, the photon pulses had a spacing of $5.5 \mu \mathrm{s}$, limited by the flight times of the heaviest fragments. Depending on other operating constraints of the facility, we received 250-570X-ray pulses per second. The photon energy was $2 \mathrm{keV}$. The single-shot $\mathrm{X}$-ray pulse energy ( $1 \mathrm{~mJ}$ on average) was measured upstream of the beamline mirrors by a gas monitor detector. The transmission was measured to be $80 \%$ at $2 \mathrm{keV}$ for the beamline configuration during user run 3 , resulting in $0.8 \mathrm{~mJ}$ on the target. The X-ray pulses were focused by a pair of Kirkpatrick-Baez mirrors, and the calculated focus size was $1.4 \mu \mathrm{m}$. The calculated upper limit of the X-ray pulse duration was $25 \mathrm{fs}$ based on the electron bunch charge of $250 \mathrm{pC}$ in the accelerator. To date, no direct measurement of the X-ray pulse duration has been carried out at the European XFEL, but recent indirect measurements suggested that the pulse duration can be as short as $10 \mathrm{fs}^{34}$. Simulations in the manuscript were carried out with $10 \mathrm{fs}$ (full-width at half-maximum) pulses. The calculated Newton plots for $20 \mathrm{fs}$ pulse duration (Extended Data Fig. 2) look very similar. The average total molecular charge is +9.2 for $10 \mathrm{fs}$ and +9.1 for $20 \mathrm{fs}$; the fraction of $\mathrm{I}^{+}+\mathrm{N}^{+}$ trajectories decreases from $30 \%$ ( $10 \mathrm{fs}$ ) to $24 \%$ ( $20 \mathrm{fs}$ ).

Experimental setup. Vapour of the target substances $\left(\mathrm{C}_{5} \mathrm{H}_{4} \mathrm{IN}\right.$ or $\left.\mathrm{C}_{4} \mathrm{H}_{3} \mathrm{IN}_{2}\right)$ was generated by heating an out-of-vacuum reservoir to $60^{\circ} \mathrm{C}$. A supersonic gas jet was formed using $500 \mathrm{mbar}$ of helium as the carrier gas, expanding the mixture into a vacuum through a nozzle of $200 \mu \mathrm{m}$ diameter, and collimating it by three skimmers and a set of adjustable slits. Gas line, nozzle and the first section of the jet vacuum chamber were also heated to $60^{\circ} \mathrm{C}$ to prevent condensation. The base pressure in the experimental chamber was retained at $1 \times 10^{-11} \mathrm{mbar}$ during operation. The jet was crossed with the $\mathrm{X}$-ray beam at $90^{\circ}$ in the centre of the reaction microscope. The spectrometer had a total length of $250 \mathrm{~mm}$, separated in two sections. At the interaction point, a high extraction field of $E=423 \mathrm{~V} \mathrm{~cm}^{-1}$ was applied (length, $86 \mathrm{~mm}$ ), whereas the remaining part was set to the same voltage as the end of the high-field region. This combination yielded a sufficient mass-over-charge resolution, and provided decent momentum resolution and keeping the total applied voltage to $<4,000 \mathrm{~V}$. The fragment ions created by the $\mathrm{X}$-ray ionization were extracted towards a time- and position-sensitive large-area microchannel plate detector (diameter, $120 \mathrm{~mm}$ ) with hexagonal delay-line position readout. The ions originating from each X-ray pulse were measured in coincidence. The analogue detector signals were recorded at $10 \mathrm{~Hz}$ with fast analogue-to-digital converters.

Reconstruction of ion momenta. The ion trajectories inside the spectrometer were reconstructed from the recorded impact times and positions, yielding the initial momentum vectors of the ions. The ion flight times depend on the mass-over-charge ratio $(\mathrm{m} / \mathrm{q})$ and thus can be used to identify the ionic species. However, the statistical distribution of a given $\mathrm{m} / \mathrm{q}$ value has a spread in time due to the ion's kinetic energy. This can cause ions with similar $m / q$ ratios to partially overlap. In the present experiment, this was the case for $\mathrm{N}^{+}$and $\mathrm{C}^{+}$in particular. In the coincidence analysis, we, therefore, omitted $\mathrm{N}^{+}$with a high initial laboratory-frame momentum directed towards the detector, that is, $\left|p_{\text {lab }, z}\right|>175$ a.u. Moreover, 3D momentum gates were applied to $\mathrm{H}^{+}, \mathrm{C}^{+}, \mathrm{N}^{+}$and $\mathrm{I}^{+}$to suppress random coincidences, restricting the laboratory-frame momentum distributions to $\left|p_{\mathrm{H}}\right|<100$ a.u., $\left|p_{\mathrm{C}}\right|<230$ a.u., $\left|p_{\mathrm{N}}\right|<300$ a.u. and $\left|p_{\mathrm{I}}\right|<480$ a.u., respectively (covering the entire momentum distributions). An additional momentum gate was applied to the sum momentum of all the measured particles, requiring $\left|p_{\text {sum }}\right|<500$ a.u.

Creation of Newton plots. Molecules in the gas phase are randomly oriented in space. In a coincidence measurement, transformation from the laboratory frame to the molecular frame can be made by selecting the emission directions of certain ions as a reference. To obtain the momentum distributions in Fig. 1, the $z$ axis of the coordinate frame was defined along the momentum of the iodine ion. The momentum of the nitrogen ion fixes the $y-z$ plane (the plane spanned by iodine and nitrogen momenta). For 2-iodopyridine, the direction of the $y$ axis was defined such that the $y$ component of the nitrogen momentum is positive. For 2 -iodopyrazine, the two nitrogen atoms were distinguished by the relative emission angle $\alpha$ between the measured nitrogen and iodine ions. Nitrogen ions with $\cos (\alpha)>-0.125$ were labelled N1 (Fig. 1), and if an N2 nitrogen was identified, the $y$ axis was mirrored. To obtain the representation shown in Fig. 3a, all the ion momenta were further normalized to the magnitude of the iodine momentum, that is, scaled by a factor of $1 /\left|p_{\mathrm{I}}\right|$ for each event. Fourfold coincidence events, where at least one hydrogen, one carbon, one nitrogen and one iodine ion were detected in coincidence, were used (Fig. 1), even though only three ions are depicted in each panel. Threefold as well as higher-order coincidences yield identical-looking Newton plots (Extended Data Fig. 1). Coincidence detection of up to eight ions was feasible, although with low statistics.
Extraction of charge-state distributions. The site-specific carbon charge-state distributions (Fig. 4) were obtained from the threefold coincidence dataset. For each carbon charge state, normalized Newton plots (analogous to Fig. 3a) were created. Fitting a sum of five 2D Gaussian functions (Fig. 3c) yields the relative abundances at each carbon site (C2-C6). Neutral fragments are not detected in the experiment, but we consider the carbon atoms to have a very low probability to stay neutral, particularly in coincidence with higher charge states of iodine. This is also consistent with the fact that extremely few neutral $\mathrm{C}$ atoms are found in the calculations.

Statistics and error bars. The 2-iodopyridine data shown in Fig. 1 were obtained within $15 \mathrm{~h}$ of data collection and those for 2 -iodopyrazine in $6 \mathrm{~h}$. The statistical validity of the data can be estimated from the number of recorded ions per figure. Figures 1 b,d and 3 a show a total of 491,986 recorded ions. Figure 1g,i shows 364,450 ions and Fig. 3 b depicts 70,068 ions in total. On close inspection of Fig. 3c, it is clear that the carbon-ion momentum distributions are only approximately described by $2 \mathrm{D}$ Gaussian functions. We, therefore, consider the fitting errors alone to underestimate the real error present in the extracted ion yields (Fig. 4). We have instead reperformed the fitting after rotating the Newton plot by $45^{\circ}$, to account for the partially asymmetric distributions, and used the difference obtained from both fits as a systematic error. This error probably somewhat overestimates the experimental uncertainty. For higher iodine charge states, a small diffuse background feature partially overlaps with the distribution of singly charged C2. We accounted for this by adding an additional 2D Gaussian to the fitting function, which is subtracted before processing the ion yield. In this way, we verified that this background does not cause the increased yield of singly charged C2 (Fig. 4b,c)

Theoretical modelling. The XMDYN framework ${ }^{26}$ follows a hybrid quantumclassical approach: neutral atoms, atomic ions and free electrons are considered classical particles, and are propagated using classical molecular dynamics. Bound electrons are treated as quantum electrons assigned to atomic orbitals, disregarding molecular states. Orbital binding energies, photoionization cross sections, and Auger and fluorescence decays rates are supplied by the ab initio XATOM toolkit ${ }^{26}$. Ionization dynamics leading to changes in the occupation numbers of the atomic orbitals are tracked in time using a Monte Carlo algorithm. All the occupied atomic orbitals of the neutral ground state are taken into account. The initial neutral equilibrium molecular geometries were obtained using the general atomic and molecular electronic structure system (GAMESS) ${ }^{35}$ at the restricted Hartree-Fock level of theory with a $3-21 \mathrm{G}^{*}$ basis set. The resulting structure of 2 -iodopyridine is in reasonable agreement with the experimental parameters ${ }^{36}$. We applied the velocity Verlet algorithm with one million time steps of one attosecond propagating the Monte Carlo trajectories up to $1 \mathrm{ps}$. Unless specified otherwise, a fluence of $7.5 \times 10^{10}$ photons $\mu \mathrm{m}^{-2}$ was employed, optimized to yield a high portion of $\mathrm{I}^{+}+\mathrm{N}^{+}$coincidences. As a consequence of the highly selective nature of the presented reaction microscope data, many more Monte Carlo realizations had to be calculated than could be used in Fig. 4 (Extended Data Table 2).

Classical over-the-barrier model in XMDYN. In XMDYN, all the charged particles interact via Coulomb forces. Atoms can exchange electrons via charge transfer according to a classical over-the-barrier model ${ }^{37}$, which yields critical distances for charge transfer that agree well with experiments ${ }^{23,38}$. The model determines electron transfer for any diatomic pair of neighbouring atoms, from donor $D$ to acceptor $A$. Electrons from any atom in the molecule can, thus, be transferred to the iodine atom. The potential experienced by the electron being transferred from $D$ to $A$ is given by

$$
V(\mathbf{r})=-\frac{Q_{D}+1}{\left|\mathbf{r}-\mathbf{r}_{D}\right|}-\frac{Q_{A}}{\left|\mathbf{r}-\mathbf{r}_{A}\right|},
$$

where $Q_{D}$ and $\mathbf{r}_{D}\left(Q_{A}\right.$ and $\left.\mathbf{r}_{A}\right)$ are the charge and position of the donor (acceptor), respectively. The top of the potential barrier along the straight line connecting $D$ and $A$ is given by

$$
V_{\mathrm{b}}=-\frac{\left(\sqrt{Q_{D}+1}+\sqrt{Q_{A}}\right)^{2}}{R},
$$

where $R=\left|\mathbf{r}_{A}-\mathbf{r}_{D}\right|$ is the donor-acceptor distance. Using first-order perturbation theory within the electrostatic monopole approximation, the energy level of the donor, $E_{D}$, is determined by the atomic orbital energy, $\varepsilon_{D}$, influenced by the electrostatic interaction with atom $A$ :

$$
E_{D}=\varepsilon_{D}-\frac{Q_{A}}{R}
$$

We perform electron transfer if the electron energy at the donor is higher than the potential barrier, that is, when the condition

$$
E_{D}>V_{\mathrm{b}}
$$

is satisfied. 
XMDYN model improvements. Prior to this work, an electron-transfer methodology also used in surface hopping ${ }^{39}$ was employed in $\mathrm{XMDYN}^{32,33}$ ensuring energy conservation after electron transfer between the atomic orbitals with different energies via a small, sudden kinetic energy transfer to the ion cores. In those cases, all the atoms had the same atomic number and the system consisted of neutral or singly charged atoms, such that the electronic configurations of two atoms typically were simply exchanged in the charge transfer, yielding no overall energy change. In the present study, atomic numbers strongly differ and the system is highly ionized; therefore, large energy differences relative to the total energy of the system can emerge between the atomic orbitals involved in an electron transfer. Modifying the ion kinetic energies to enforce strict energy conservation, thus, leads to strong artifacts in the Newton plots (Extended Data Fig. 7A,B). We, therefore, disregarded the atomic orbital energy differences and changes in the potential energies in the current charge-transfer scheme, and did not perform any sudden change in ion kinetic energies. The obtained Newton plots in Fig. 1 and Extended Data Fig. 7C,D are in very good agreement with the experimental results.

A further modification introduced in XMDYN improved the agreement between the calculated charge-state distributions and the experimental data (Fig. 4), particularly the average total charges. Direct transfer of electrons from other atoms into the $\mathrm{M}$ and $\mathrm{N}$ shells of iodine needed to be excluded (Extended Data Fig. 8). Allowing such electron transfer neglected the Auger lifetimes (1.1 and $13.4 \mathrm{fs}$ ) of holes in the $\mathrm{M}$ and $\mathrm{N}$ shells of iodine, which predominantly absorb X-ray photons at $2 \mathrm{keV}$ photon energy (Extended Data Table 1). Moreover, the transferred electron no longer occupies the outermost (highest quantum number) acceptor atomic orbital with a vacancy. Instead, the electron is put into the orbital with a vacancy at the acceptor atom that is the most similar in energy to the donor-atom orbital originally occupied by the electron. This approach is based on quantum mechanical principles. Extended Data Fig. 8 illustrates this improvement.

Ion momenta in experiment and theory. The calculated ion momenta (Fig. 1) are overestimated by about $60 \%$ for $\mathrm{I}^{+}, 40 \%$ for $\mathrm{C}^{+}, 30 \%$ for $\mathrm{N}^{+}$and $20 \%$ for $\mathrm{H}^{+}$with respect to the experimentally measured values. We consider this to be a consequence of the lack of chemical bonds in the simulation, which give rise to a force naturally working against the Coulomb explosion into atomic fragments. An earlier study found that after X-ray multiphoton ionization, $\mathrm{C}_{60}$ with $\sim 13$ positive charges does not undergo instantaneous explosion, but takes time to break up into smaller fragments with still-existing chemical bonds ${ }^{33}$. In that case, the XMDYN simulation included charge transfer as well as chemical bonds, which strongly reduced the maximum $\mathrm{C}$-atom displacement compared with a simulation without chemical effects. We have recently implemented a generic reactive force field describing bond breaking and bond formation for molecules other than $\mathrm{C}_{60}$ in XMDYN, but although first-test calculations on light-atom systems were successful, the force field cannot yet describe heavy atoms such as iodine. In the present study, the average charge on each of the atoms is considerably higher than in the case of $\mathrm{C}_{60}$, and we do not have evidence for a significant formation of intermediate molecular fragments. Therefore, a large part of the total electrostatic energy is spent on breaking the chemical bonds, diminishing the Coulomb energy being turned into kinetic energy, and thus reducing the momenta of atomic ions. Based on tabulated bond dissociation energies, the sum of all the binding energies in 2-iodopyridine would be $54 \mathrm{eV}$. The highly ionized states in the present study are, however, expected to have smaller binding energies compared with the neutral ground state. For the coincidence channels involving higher charges, the overestimation of the iodine momentum becomes smaller (about $50 \%$ for $\mathrm{I}^{4+}+\mathrm{N}^{2+}$ and $40 \%$ for $\mathrm{I}^{6+}+\mathrm{N}^{2+}$ versus $60 \%$ for $\left.\mathrm{I}^{+}+\mathrm{N}^{+}\right)$. This indicates that the missing chemical bonds become less important at higher total charges, for which the potential energy surfaces start to resemble a pure Coulomb repulsion without bound potentials. This is in line with another study ${ }^{9}$ in which a model covering only Coulombic forces and no chemical bonds overestimates the fragment-ion velocities for low charges, but achieves an increasingly adequate description for higher charges. Furthermore, another study ${ }^{40}$ suggested bound potentials as a reason for the experimental kinetic-energy release of $\mathrm{CH}_{3} \mathrm{I}$ fragmenting into $\mathrm{CH}_{3}^{+}+\mathrm{I}^{q+}(q \leq 3)$ being lower than the one calculated for pure Coulomb potentials.

\section{Data availability}

Source data are available for this paper. Data recorded for the experiment at the European XFEL are available at https://doi.org/10.22003/xfel.eu-data-002159-00. All other data that support the plots within this paper and other findings of this study are available from the corresponding authors upon reasonable request.

\section{Code availability}

The data-analysis code is available from the corresponding authors upon reasonable request. An executable of XMDYN is available at https://www.desy. de/ xraypac/license.html.

\section{References}

32. Kumagai, Y. et al. Radiation-induced chemical dynamics in Ar clusters exposed to strong X-ray pulses. Phys. Rev. Lett. 120, 223201 (2018).

33. Berrah, N. et al. Femtosecond-resolved observation of the fragmentation of buckminsterfullerene following X-ray multiphoton ionization. Nat. Phys. 15, 1279-1283 (2019)

34. Khubbutdinov, R. et al. High spatial coherence and short pulse duration revealed by the Hanbury Brown and Twiss interferometry at the European XFEL. Struct. Dyn. 8, 044305 (2021).

35. Schmidt, M. W. et al. General atomic and molecular electronic structure system. J. Comput. Chem. 14, 1347-1363 (1993).

36. Sortur, V., Yenagi, J. \& Tonannavar, J. Vibrational assignments of 2-iodopyridine. Spectrochim. Acta A Mol. Biomol. Spectrosc. 69, 604-611 (2008).

37. Ryufuku, H., Sasaki, K. \& Watanabe, T. Oscillatory behavior of charge transfer cross sections as a function of the charge of projectiles in low-energy collisions. Phys. Rev. A 21, 745-750 (1980).

38. Boll, R. et al. Charge transfer in dissociating iodomethane and fluoromethane molecules ionized by intense femtosecond X-ray pulses. Struct. Dyn. 3, 043207 (2016).

39. Subotnik, J. E. et al. Understanding the surface hopping view of electronic transitions and decoherence. Annu. Rev. Phys. Chem. 67, 387-417 (2016).

40. Corrales, M. E. et al. Velocity map imaging and theoretical study of the Coulomb explosion of $\mathrm{Ch}_{3} \mathrm{I}$ under intense femtosecond IR pulses. J. Phys. Chem. A 116, 2669-2677 (2012).

\section{Acknowledgements}

We acknowledge European XFEL in Schenefeld, Germany, for the provision of XFEL beam time at the SQS instrument and would like to thank the staff for their assistance. The reaction microscope and its implementation at SQS are funded by the BMBF. R.D., K.F., S.G., M.H., M.I., G.N., M.S., I.V.-P., and M.W. acknowledge support from Deutsche Forschungsgemeinschaft (DFG) via Sonderforschungsbereich 1319 (ELCH). M.I., V.M., and P.H.S. acknowledge funding from the Volkswagen Foundation within a Peter Paul Ewald Fellowship. B.R., K.K., M.M. and R.S. were supported by the Cluster of Excellence 'Advanced Imaging of Matter' of the DFG-EXC 2056 and project ID 390715994. M.M. and T.M. acknowledge support by the DFG - SFB-925 - project 170620586. S.E. acknowledges funding of DFG through Priority Programme SPP 1840 QUTIF. L.I., X.L. and R.S. acknowledge support from the Chemical Sciences, Geosciences, and Biosciences Division, Office of Basic Energy Sciences, Office of Science, US Department of Energy, via grant no. DE-SC0019451; D.R. and A.R. acknowledge support by grant no. DE-SC0020276 from the same funding agency. R.J. is supported by the Helmholtz-OCPC Postdoc Program. P.W. is supported by the US Department of Energy, Office of Science, Office of Basic Energy Sciences, under contract no. DE-AC02-76SF00515. A.H. acknowledges funding of the Studienstiftung des Deutschen Volkes.

\section{Author contributions}

R.B. proposed the study. T.J. analysed the data. J.M.S. carried out the calculations, with support from R.S., Z.J., B.R., S.-K.S., M.M.A., L.I. and R.J. The experiment was carried out by R.B., J.M.S., K.F., M.S., S.E., B.E., M.I., K.K., X.L., T.M., C.P., D.E.R., D.R., J.S., P.H.S., N.S., F.T., P.W., M.M. and T.J. after the experiment was previously prepared and commissioned by G.K., N.A., T.M.B., A.D.F., L.I., S.G., P.G., A.H., M.H., C.J., M. Kircher, M. Kunitski, S.M., N.M., J.M., V.M., G.N., Y.O., A.P., N.R., J.R., I.S., L.P.H.S., D.T., I.V.-P., M.W., R.W. and P.Z. The REMI experimental chamber was designed and built by G.K., T.J., M.S., R.D. and L.P.H.S., with contributions from the whole University of Frankfurt group. R.B., T.J., J.M.S. and R.S. wrote the manuscript, after in-depth discussions with R.D., D.R., S.-K.S., A.R. and M.M., with input from all the authors.

\section{Funding}

Open access funding provided by Deutsches Elektronen-Synchrotron (DESY).

\section{Competing interests}

The authors declare no competing interests.

\section{Additional information}

Extended data is available for this paper at https://doi.org/10.1038/s41567-022-01507-0. Supplementary information The online version contains supplementary material available at https://doi.org/10.1038/s41567-022-01507-0.

Correspondence and requests for materials should be addressed to Rebecca Boll, Robin Santra or Till Jahnke.

Peer review information Nature Physics thanks the anonymous reviewers for their contribution to the peer review of this work

Reprints and permissions information is available at www.nature.com/reprints. 

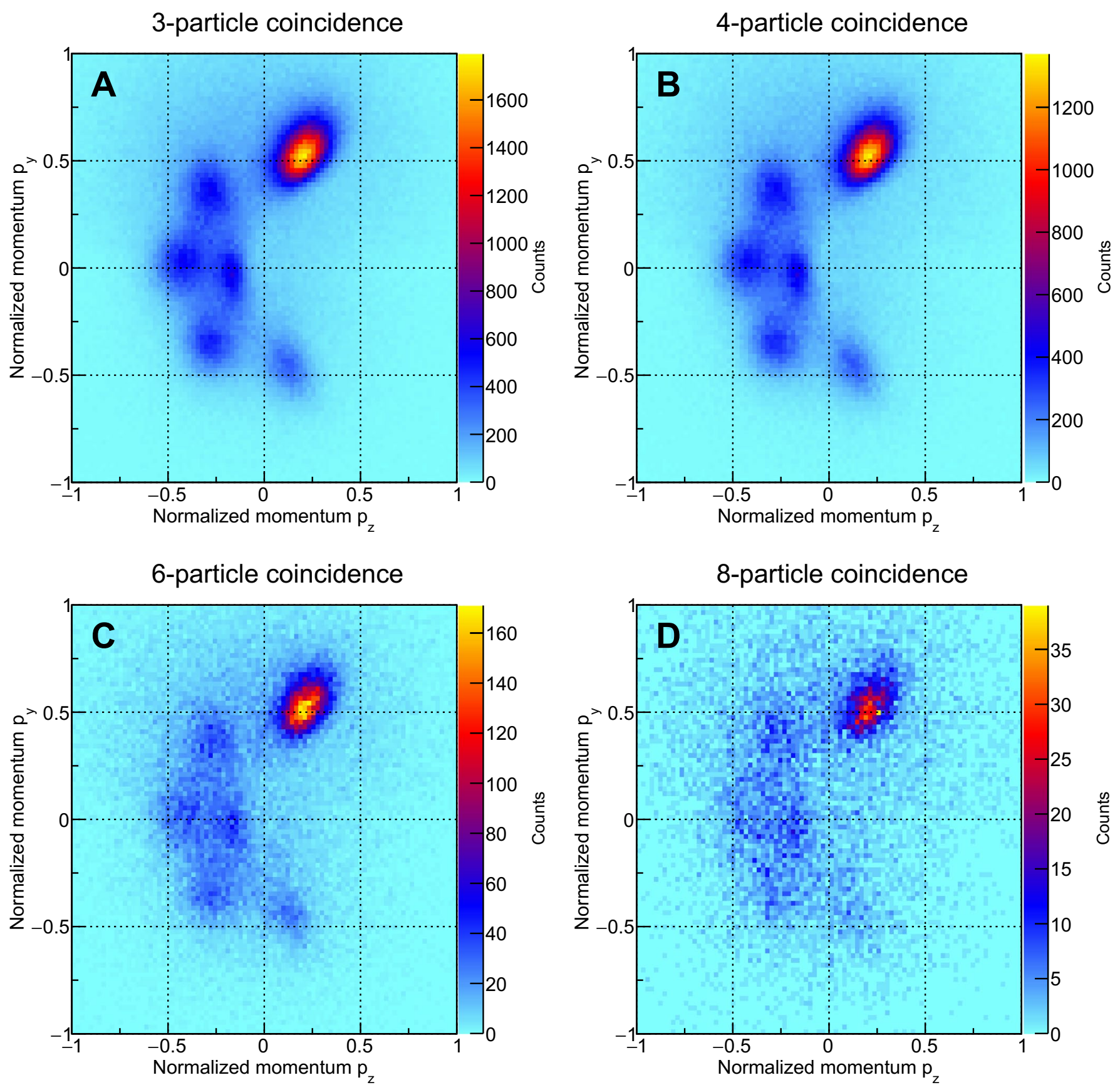

Extended Data Fig. 1 | Newton plots for higher coincidence conditions. Newton plots for higher coincidence conditions. (A) Three-fold $\left[I^{+}+\mathrm{N}^{+}+\mathrm{C}^{+}\right]$, (B) four-fold $\left[\mathrm{I}^{+}+\mathrm{N}^{+}+\mathrm{C}^{+}+\mathrm{H}^{+}\right],(C)$ six-fold $\left[\mathrm{I}^{+}+\mathrm{N}^{+}+3 \times \mathrm{C}^{+}+\mathrm{H}^{+}\right]$, and (D) eight-fold $\left[\mathrm{I}^{+}+\mathrm{N}^{+}+3 \times \mathrm{C}^{+}+3 \times \mathrm{H}^{+}\right]$coincidence analysis. Note that, in contrast to Fig. 1, the ion intensity is plotted as counts. Therefore, the nitrogen ion appears overestimated by a factor of 5 as compared to the carbon ions. In all cases, one nitrogen ion was detected and is plotted, while only one of the five different carbon ions is plotted (even in cases where more than one carbon ion was detected). 

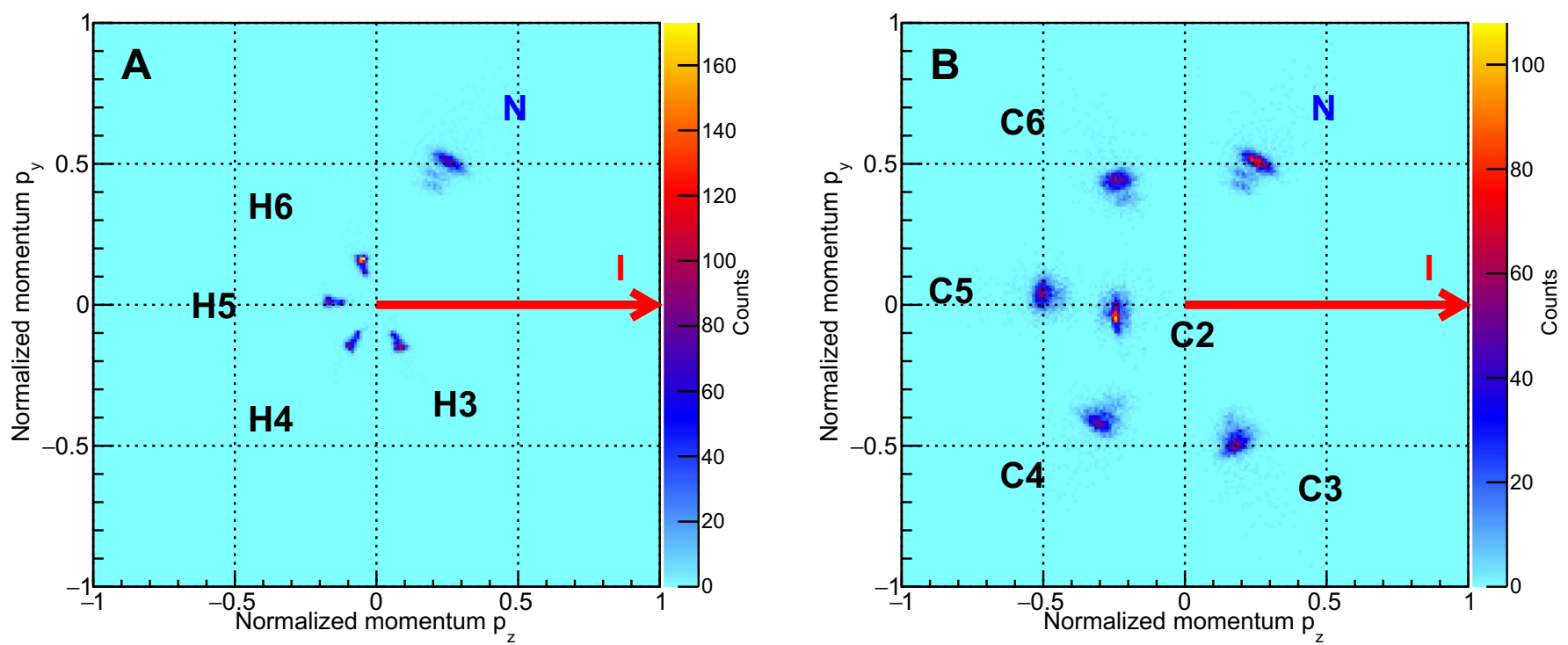

Extended Data Fig. 2 | Pulse duration dependence. Pulse duration dependence. Newton plots from XMDYN for 20fs FWHM pulse duration and a fluence of $7.5 \times 10^{10}$ photons $/ \mu \mathrm{m}^{2}$. Results are shown for a coincident detection of $\mathrm{I}^{+}+\mathrm{N}^{+}$and all occurring $\mathrm{H}^{+}$ions $(\mathrm{A})$, and $\mathrm{I}^{+}+\mathrm{N}^{+}$and all occurring $\mathrm{C}^{+}$ions (B). The magnitudes of all ion momenta are normalized such that the iodine momentum equals $1\left(p_{l_{2}}=1\right.$ ). This figure is the $20 \mathrm{fs}$ counterpart to the $10 \mathrm{fs}$ results in the second row of Extended Data Fig. 7 and the calculations shown in Fig. 1. 

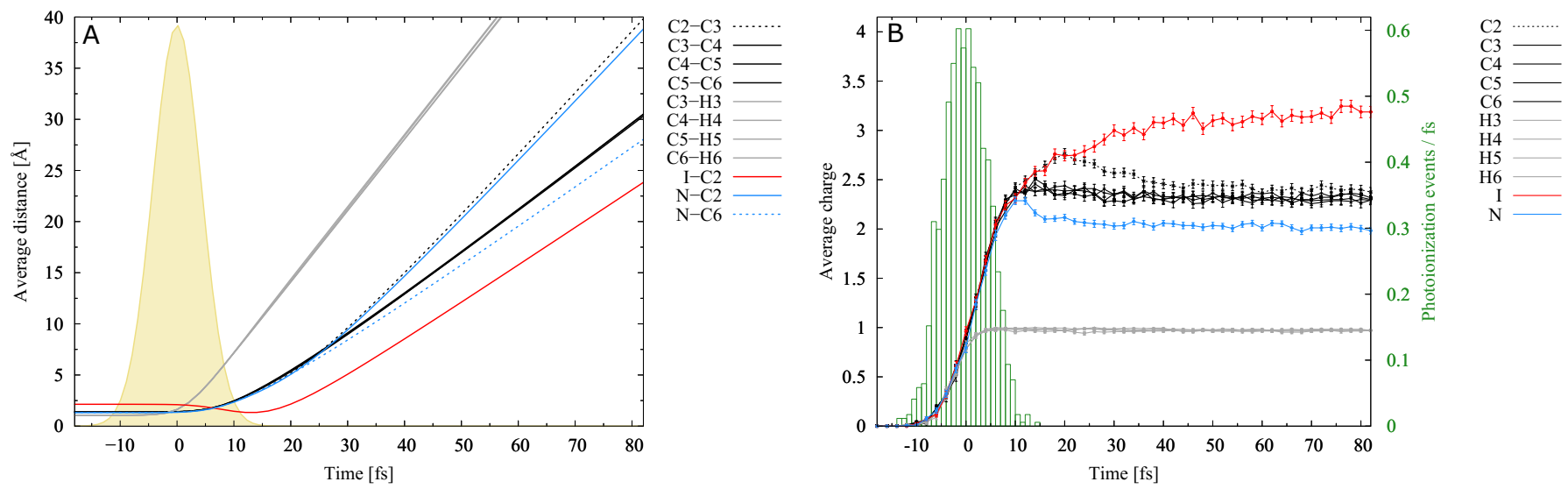

Extended Data Fig. 3 | Time-dependent geometry and charge evolution of 2-iodopyridine molecules for $\mathbf{I}^{4+}+\mathrm{N}^{2+}$ coincidences. Time-dependent geometry and charge evolution of 2-iodopyridine molecules for $\mathrm{I}^{4+}+\mathrm{N}^{2+}$ coincidences. (A) Average interatomic distances and $\mathrm{X}$-ray pulse shape (10fs FWHM, yellow); (B) average charge on each atom and average number of photoionizations per molecule and femtosecond (green bars). The error bars in (B) correspond to the standard error of the mean; in (A), those errors are smaller than the data point markers. In comparison to Fig. 2, the structural changes start earlier and have higher velocity. The I-C2 distance minimum is reached immediately after the end of the pulse, and the hydrogen charges rise rapidly until nearly all hydrogens are ionized $5 f s$ after the pulse peak. A subset of the trajectories resulting in $\mathrm{I}^{4+}+\mathrm{N}^{2+}$ ions at the end of the propagation time, calculated with a fluence of $7.5 \times 10^{10}$ photons $/ \mu \mathrm{m}^{2}$, was used for this figure. 

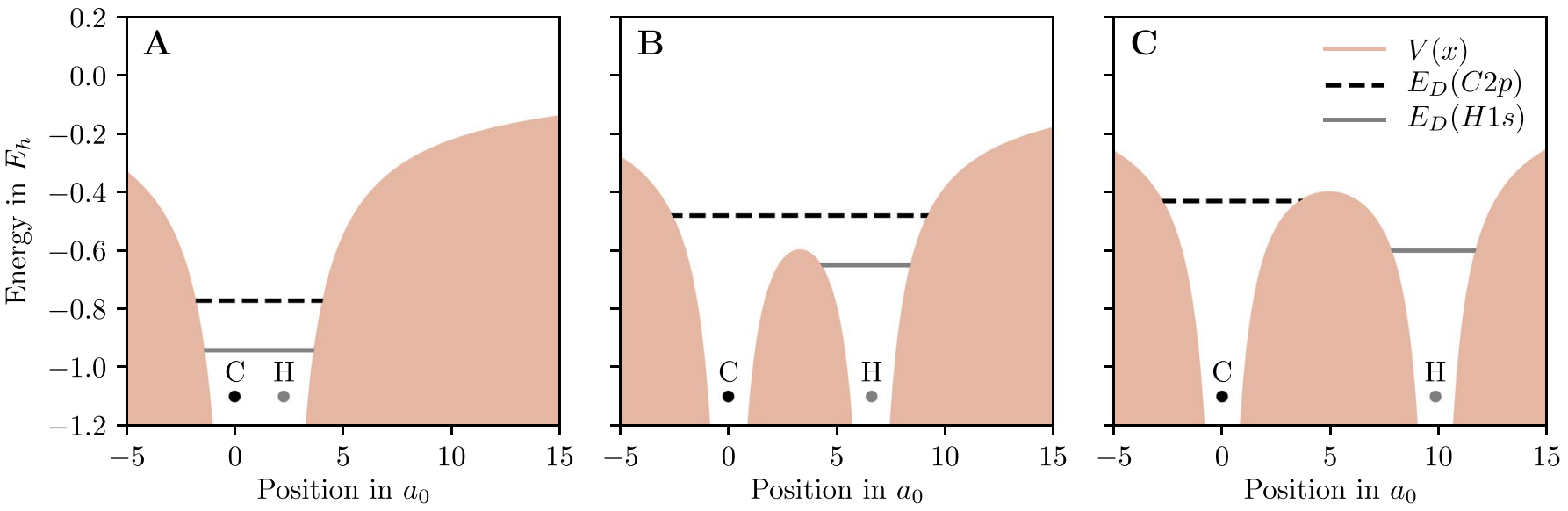

Extended Data Fig. 4 | Time-dependent potential barriers. Time-dependent potential barriers between $\mathrm{C} 3$ and $\mathrm{H} 3$ at $t=0 \mathrm{fs}(\mathrm{A}), 10 \mathrm{fs}(\mathrm{B})$, and $14 \mathrm{fs}(\mathrm{C})$, where one of the atoms carries a charge of +1 and the other one is neutral, respectively. The donor energy levels $E_{D}(\mathrm{C} 2 p)$ and $E_{D}(\mathrm{H} 1 \mathrm{~s})$ are based on the atomic orbital energies, influenced by the electrostatic interaction with the other atom, using Eq. (3) in Methods. The potential shown, $V(x)=-\frac{1}{|x|}-\frac{1}{|x-R|}$ (where $R$ is the average C3-H3 distance at time $t$ (see Fig. 2C)), corresponds to a charge of +1 on both atomic sites, as the electron on the neutral atom considered for the charge transfer does not feel its own shielding of the nuclear charge. Filled areas indicate forbidden regions (no tunneling included in the model). For $t<10 \mathrm{fs}(\mathrm{A})$, electron transfer is possible in both directions; for $10<t<14 \mathrm{fs}$ (B), electron transfer is only possible from $\mathrm{C}_{\text {to }} \mathrm{H}^{+}$, but not from $\mathrm{H}$ to $\mathrm{C}^{+}$; for $t>14 \mathrm{fs}(\mathrm{C})$, the potential barrier exceeds both energy levels, and no electron transfer can occur. The one-way electron transfer between $10 \mathrm{fs}$ and $14 \mathrm{fs}$ explains the decrease of the average hydrogen charges in the same time period in Fig. 2D. 


\section{NATURE PHYSICS}
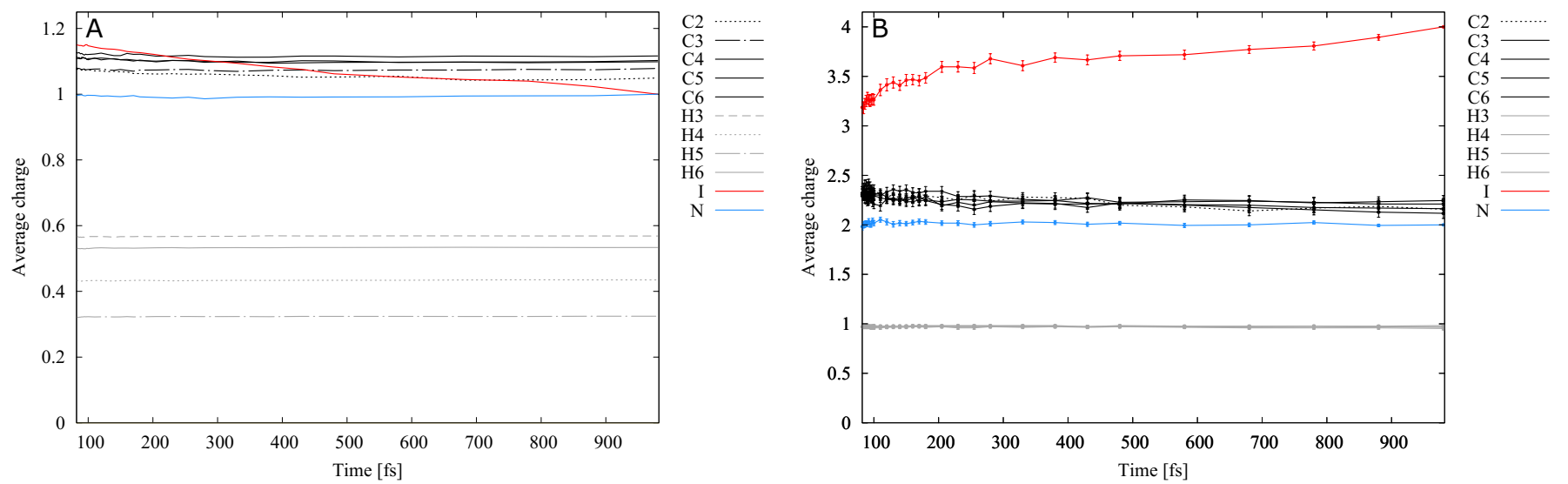

Extended Data Fig. 5 | Late-time charge evolution. Late-time charge evolution for 2 -iodopyridine for $\left.(A)\right|^{+}+\mathrm{N}^{+}$coincidences (continuation of Fig. 2D), and (B) $\mathrm{I}^{4+}+\mathrm{N}^{2+}$ coincidences (continuation of Extended Data Fig. 3B). The error bars in (B) correspond to the standard error of the mean, in (A), those errors are smaller than the data point markers. The final charge states, in particular of iodine, are only reached at the end of the propagation time, after about 1 ps, which can be understood as follows: photo- and Auger electrons are treated as classical particles in XMDYN. If they are captured in the attractive potential created by all charged ions, they are no longer considered free particles, but delocalized electrons. If their kinetic energy is lower than the electrostatic attraction towards a given ion, they are trapped in its field and the ion's charge is correspondingly reduced by -1 . The decrease/increase in (A)/(B) is caused by an increase/decrease in the average number of electrons trapped around iodine. A possible reason for the increase of the iodine charge in (B) is autoionization-like events between two trapped electrons, or between a delocalized electron and a trapped one, where one trapped electron receives enough energy to leave the field around the ion. 

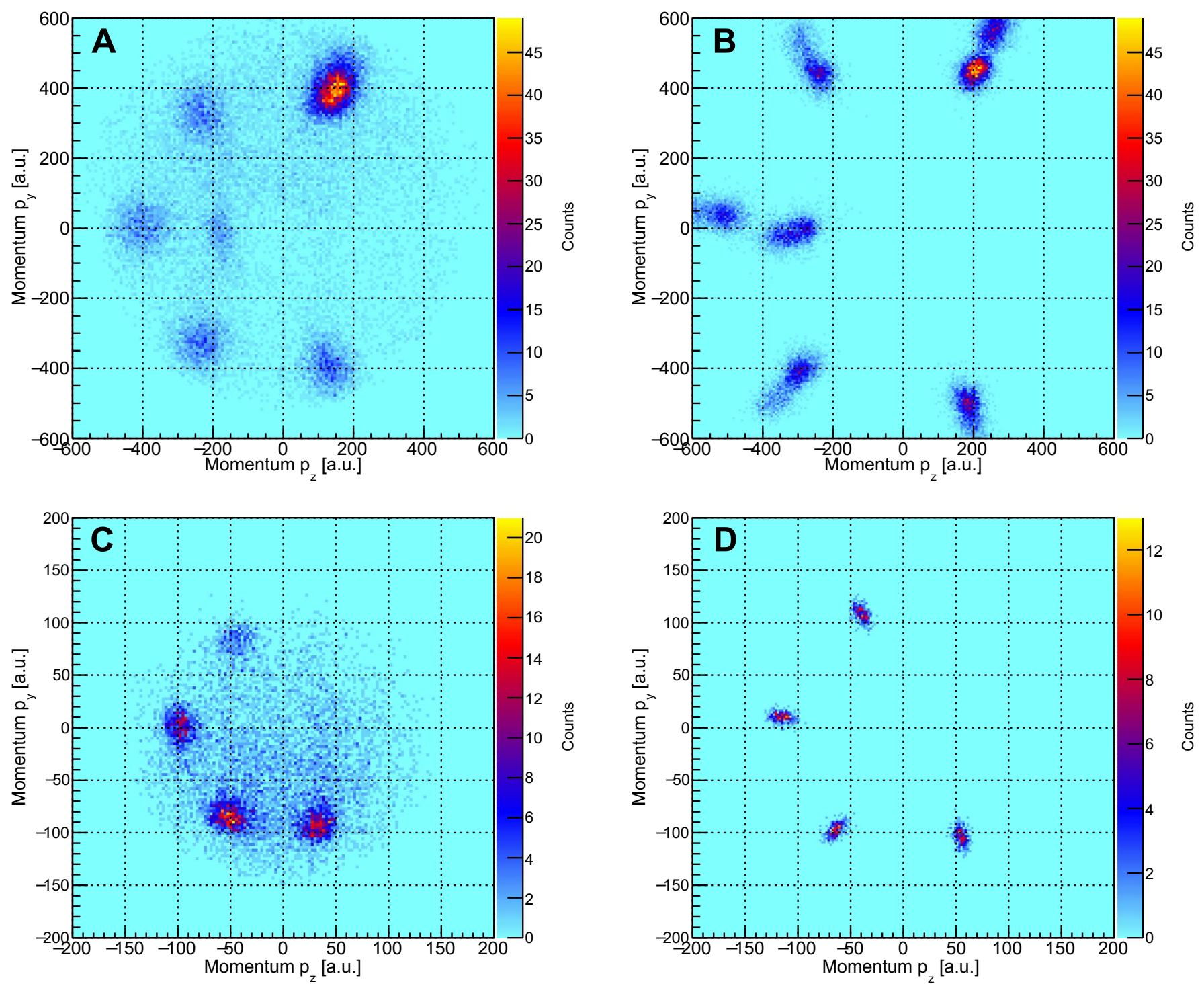

Extended Data Fig. 6 | Newton plots for higher charge states. Newton plots for higher charge states. Experimental (A), (C) and calculated (B), (D) Newton plots for coincidences of $\mathrm{I}^{4+}+\mathrm{N}^{2+}+\mathrm{C}^{3+}(A),(B)$, and $\mathrm{I}^{6+}+\mathrm{N}^{2+}+\mathrm{H}^{+}(C),(D)$. The channels with higher total charge look qualitatively similar to Fig. 1, but the absolute momenta increase and the individual atoms become more separated. The fluences used in the calculations are $1.80 \times 10^{11}(B)$ and $2.30 \times 10^{11}$ photons $/ \mu \mathrm{m}^{2}(\mathrm{D})$. 

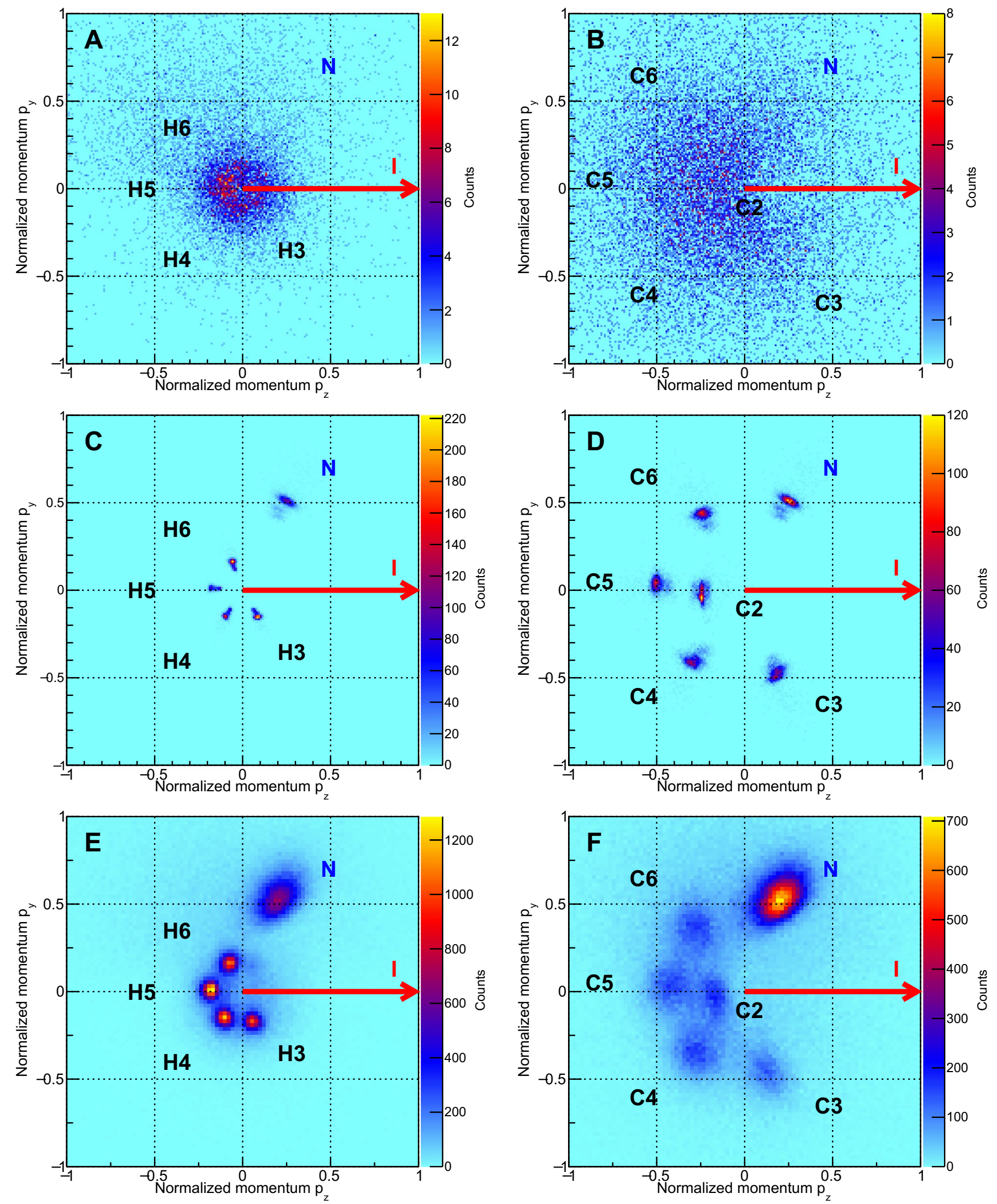

Extended Data Fig. 7 | Newton plots with and without sudden kinetic energy transfer to the ion cores. Newton plots with and without sudden kinetic energy transfer to the ion cores. Comparison of calculated $I^{+}+N^{+}$Newton plots from XMDYN (A)-(D) to experimental data $(E, F)$. Shown are $H^{+}(A$, $C, E)$ and $C^{+}$ion momenta $(B, D, F)$. The results $(A, B)$ were obtained with the old scheme (see text) of treating orbital energy differences in chargetransfer events (Methods). In a revised approach, no sudden change of ion kinetic energies was performed (C, D). The magnitudes of all ion momenta are normalized such that the iodine momentum equals $1\left(p_{l_{z}}=1\right)$. 

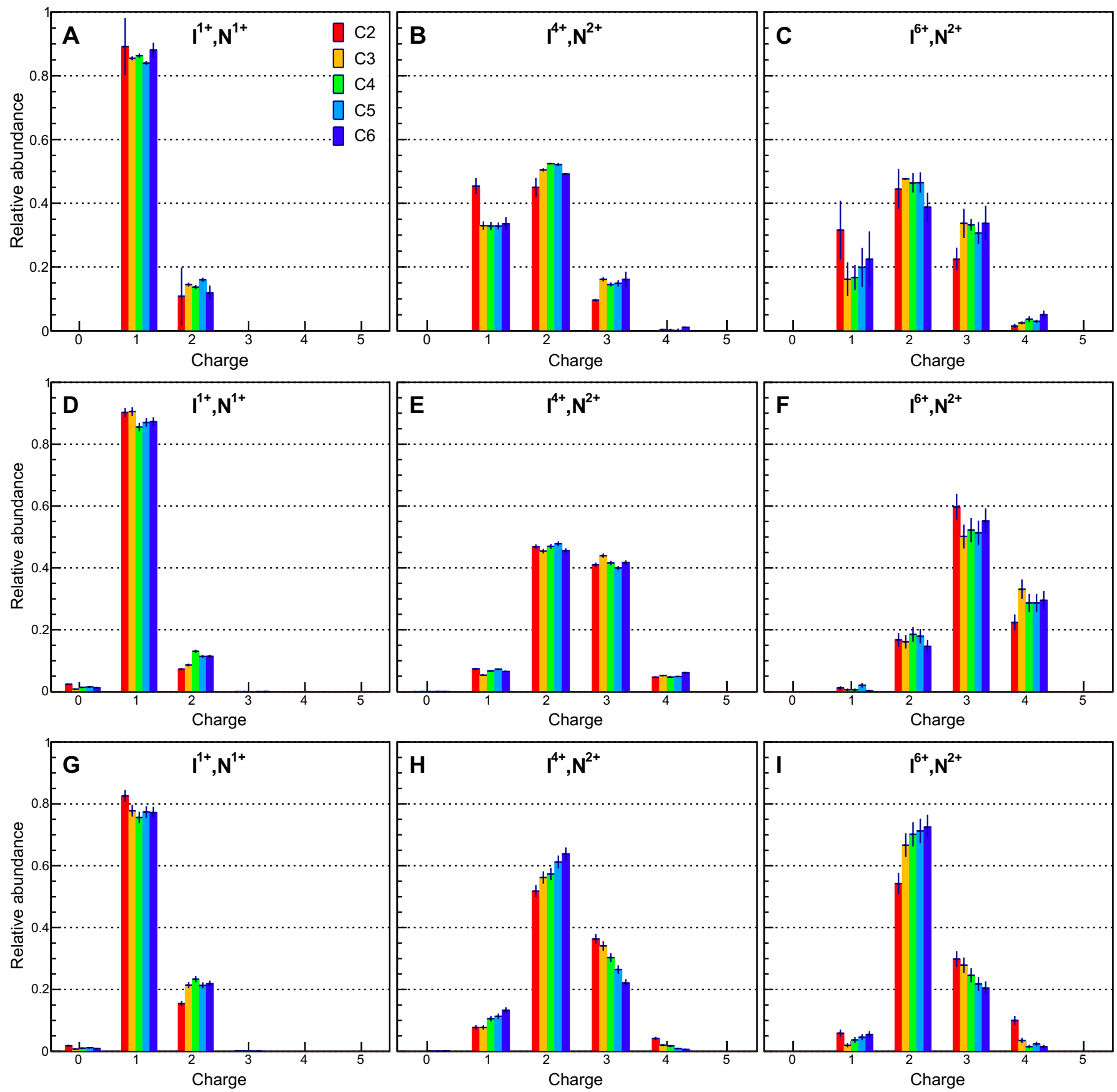

Extended Data Fig. 8 | Charge-state distributions of carbon atoms. Charge-state distributions of carbon atoms, for different coincidence channels of 2-iodopyridine. The experimental ion yields (A)-(C) are obtained by fitting a 2D Gaussian to each peak in the respective Newton plots (see Fig. 3). The experimental error bars represent an estimate of the systematic error introduced in the fitting (Methods). Note that the experiment is insensitive to neutral fragments. Theory results $(D)-(I)$ are shown with the statistical error derived from the finite number of trajectories. The fluences used in the calculations are $0.75 \times 10^{11}(\mathrm{D}),(\mathrm{G}), 1.80 \times 10^{11}(\mathrm{E}),(\mathrm{H})$, and $2.30 \times 10^{11}$ photons $/ \mu \mathrm{m}^{2}(\mathrm{~F})$, (I). Panels (D)-(F) show the results obtained from our initial model, i.e., when all orbitals of iodine with vacancies were considered. In a model refinement, electron transfer into the iodine $\mathrm{M}$ - and $\mathrm{N}$-shells was excluded (G)-(I). 


\begin{tabular}{cccccc}
\hline \hline trajectories & av. photoionizations & $\mathrm{C}$ & $\mathrm{H}$ & $\mathrm{I}$ & $\mathrm{N}$ \\
\hline all 15000 & $3.44 \pm 0.02$ & $6.6 \%$ & $0.0 \%$ & $91.1 \%$ & $2.4 \%$ \\
$4560 \mathrm{I}^{+}+\mathrm{N}^{+}$ & $3.32 \pm 0.02$ & $7.1 \%$ & $0.0 \%$ & $90.6 \%$ & $2.3 \%$ \\
$171 \mathrm{I}^{4+}+\mathrm{N}^{2+}$ & $7.00 \pm 0.10$ & $4.3 \%$ & $0.0 \%$ & $94.2 \%$ & $1.5 \%$ \\
\hline \hline
\end{tabular}

Extended Data Table 1 | Average number and atom-specific photoionizations in XMDYN. Average number and element-specific photoionizations in XMDYN for $2 \mathrm{keV}$ photon energy, $10 \mathrm{fs}$ pulse duration and a fluence of $7.5 \times 10^{10}$ photons $/ \mu \mathrm{m}^{2}$. The value for $\mathrm{C}$ is the sum of all five atoms. The I $3 d$ subshell accounts for the largest share of photoionizations, followed by the I $3 p$ subshell. Selecting the $\mathrm{I}^{+}+\mathrm{N}^{+}$trajectories leads to almost no difference compared to all trajectories. Selecting the $\mathrm{I}^{4+}+\mathrm{N}^{2+}$ trajectories on the other hand implies selecting cases where seven ionization events have occurred on average, which are found to have an even higher probability to take place at the iodine site, compared to the three photoionizations on average in all trajectories. 


\begin{tabular}{cccccr}
\hline \hline figure & coincidence & fluence $\left[\mathrm{ph} / \mu \mathrm{m}^{2}\right]$ & coinc. traj. & sum & fraction \\
\hline Fig. 4D \& Extended Data Fig. 8G & $\mathrm{I}^{+}+\mathrm{N}^{+}$ & $7.5 \times 10^{10}$ & 2212 & 15000 & $15 \%$ \\
Fig. 4E \& Extended Data Fig. 8H & $\mathrm{I}^{4+}+\mathrm{N}^{2+}$ & $1.8 \times 10^{11}$ & 1429 & 30000 & $5 \%$ \\
Fig. 4F \& Extended Data Fig. 8I & $\mathrm{I}^{6+}+\mathrm{N}^{2+}$ & $2.3 \times 10^{11}$ & 459 & 45000 & $1 \%$ \\
Extended Data Fig. 8D & $\mathrm{I}^{+}+\mathrm{N}^{+}$ & $7.5 \times 10^{10}$ & 4560 & 15000 & $30 \%$ \\
Extended Data Fig. 8E & $\mathrm{I}^{4+}+\mathrm{N}^{2+}$ & $1.8 \times 10^{11}$ & 8385 & 75000 & $11 \%$ \\
Extended Data Fig. 8F & $\mathrm{I}^{6+}+\mathrm{N}^{2+}$ & $2.3 \times 10^{11}$ & 335 & 45000 & $1 \%$ \\
\hline
\end{tabular}

Extended Data Table 2 | Fluences and numbers of XMDYN Monte Carlo trajectories. Fluences and numbers of XMDYN Monte Carlo trajectories underlying Fig. 4 and Extended Data Fig. 8. Given are the sums of all calculated trajectories, determined by the need to obtain a sufficiently small statistical error (normalized square root of the number of trajectories with the carbon atom in the respective final charge state) in Fig. 4, as well as the number of trajectories meeting the respective coincidence criterion (coinc. traj.), and the corresponding fraction. 